\title{
Interplay of coarsening, aging, and stress hardening impacting the creep behavior of a colloidal gel
}

\author{
D. C. E. Calzolari, I. Bischofberger, ${ }^{\text {a) }}$ F. Nazzani, and V. Trappe ${ }^{\text {b) }}$ \\ Department of Physics, University of Fribourg, CH-1700 Fribourg, Switzerland
}

(Received 15 November 2016; final revision received 20 May 2017; published 28 June 2017)

\begin{abstract}
We explore the dynamical and mechanical characteristics of an evolving gel in diffusing wave spectroscopy (DWS) and rheometry, aiming to assess how the gel evolution impacts the creep response of the system. Our gel is formed by inducing the aggregation of thermosensitive colloids by a variation in temperature. We find experimental evidence that the long time evolution of this gel is due to two distinct processes: A coarsening process that involves the incorporation of mobile particles into the network structure and an aging process that triggers intermittent rearrangement events. While coarsening is the main process governing the evolution of the elastic properties of the gel, aging is the process determining structural relaxation. The combination of both processes in addition to stress hardening governs the creep behavior of the gel, a creep behavior that is determined by three distinct contributions: an instantaneous elastic, a delayed elastic, and a loss contribution. The systematic investigation of these contributions in recovery experiments provides evidence that losses and delayed elastic storage have a common origin, both being due to intermittent local structural relaxation events. (C) 2017 The Society of Rheology.

[http://dx.doi.org/10.1122/1.4986465]
\end{abstract}

\section{INTRODUCTION}

Amorphous solids are ubiquitous in nature and technology, ranging from granular packings to molecular glasses [1]. The amorphous state is in general a nonequilibrium state, which makes the properties of amorphous solids difficult to predict, even more so as they are often evolving in time. In colloidal sciences, the two prominent examples of amorphous solids are colloidal glasses and colloidal gels [2]. Colloidal glasses are formed at fairly high particle volume fractions and the conditions for dynamic arrest are solely set by the formation of cages in which the particles are permanently trapped. In contrast, the formation of amorphous solids at lower particle volume fractions requires both the formation of spacespanning networks and the local arrest of the dynamics of the particles within the network. The pathways to the formation of such network can be very diverse, depending on the particle volume fraction, and the magnitude and range of the attractive particle potential [3,4]. Moreover, the highly heterogeneous configuration of gels precludes that each particle experiences the same trapping conditions on the local scale as this is normally the case in colloidal glasses. The particles on the surface of a coarse network will be able to escape more easily out of the potential wells of their nearest neighbors than particles that are buried deep inside the network [5]. These intrinsic structural and dynamical heterogeneities can lead to coarsening of the gel structure over very long times. Further adding to the complexity of the temporal evolution of gels is the gel formation itself. Starting from a fully dispersed

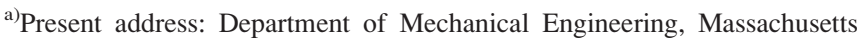
Institute of Technology, Cambridge, MA 02139.

b) Author to whom correspondence should be addressed; electronic mail: veronique.Trappe@unifr.ch
}

state, the colloidal system undergoes a sol-gel transition via aggregation or phase separation [2,3,6-13]. Beyond the gel point free particles and/or freely diffusing clusters may still exist and their gradual incorporation into the network will further contribute to the development of the structural, mechanical, and dynamical properties of the system. Indeed, bimodal distributions of particle mobilities have been observed in moderately concentrated suspensions of attractive colloids near the gel transition [14-17] and the gradual incorporation of these mobile constituents has been associated with the temporal evolution of the elasticity of the gel [5].

In this work, we address how the evolution of a gel impacts its creep behavior. Creep deformation is a rather general feature of solid materials [18-21]; it occurs when the system is subjected to a moderate constant stress. The initial response to such imposed stress is an instantaneous strain that reflects the elasticity of the system. In the following, we then observe primary creep: the strain slowly increases in time at a rate that decreases in time. In metals such creep has been extensively studied and it is generally associated to stress hardening that in turn correlates with an increase of the density of dislocations [22,23]. Our understanding of creep of amorphous solids, however, remains far from complete. In soft materials creep is ubiquitous [24-28] and of particular interest to the phenomenon of delayed yielding [26,29-33]. Upon applying a stress that is only marginally larger than the static yield stress soft matter systems typically exhibit a period of primary creep before starting to yield. To what extent we can predict delayed yielding from the creep behavior of a material and how the aging characteristics of the system may impact the creep behavior are issues that are still far from being fully understood.

In this contribution, we address these questions for a colloidal gel, focusing in particular on the range of applied 
stresses below the onset of yielding. In a first step, we investigate the evolving characteristics of a gel formed by thermosensitive particles at a particle volume fraction of 0.185 using diffusing wave spectroscopy (DWS) and oscillatory shear experiments. Both elasticity and structural relaxation time are found to evolve with sample age. Experimental evidence obtained by subjecting the gel to a constant stress indicates that the evolution of elasticity and relaxation originate from, respectively, the incorporation of mobile particles into the gel structure and intermittent events involving structural rearrangements over larger length scales. By systematically determining the recoverable strain at different time intervals, we find that creep involves not only losses but also considerable delayed elastic storage, in addition to an instantaneous elastic contribution. We show that we can fully account for the time dependences of all three contributions, when they are obtained at sufficiently low stresses, by considering (a) the age dependence of the elasticity and relaxation time of the quiescent gel and (b) a stress-hardening effect. Distinct scaling characteristics of the stress dependent loss contributions over a wider range of stresses suggest that upon increasing the stress toward the yield stress the rate of restructuring events remains the same, while the size of the rearranged zones per event increases.

\section{MATERIALS AND METHODS}

\section{A. Sample characteristics}

Our colloidal system consists of polystyrene (PS) particles coated with a thin layer of poly-N-isopropyl acrylamide (PNiPAM). These particles are synthesized by emulsion polymerization following a protocol similar to that described for the synthesis of "core-latex" in [34]. $\mathrm{N}$-isopropyl acrylamide NiPAM (23.4 g, Aldrich) and sodium dodecylsulfate SDS (0.2 g, Merck) are dissolved in $525 \mathrm{~g}$ Millipore water. Dissolved oxygen is evacuated by continuously bubbling nitrogen through the solution for $30 \mathrm{~min}$. After addition of styrene $\left(142.4 \mathrm{~g}\right.$, Fluka), the mixture is heated to $80^{\circ} \mathrm{C}$ under stirring while the bubbling of nitrogen is maintained. The initiator potassium peroxodisulfate KPS ( $0.35 \mathrm{~g}$, Merck) dissolved in $15.4 \mathrm{~g}$ Millipore water is then added and the reaction is allowed to proceed for $6 \mathrm{~h}$. For purification, the suspension is repeatedly centrifuged and the supernatant exchanged with Millipore water. In a final step, the suspending medium is adjusted to a composition of $\mathrm{H}_{2} \mathrm{O} / \mathrm{D}_{2} \mathrm{O}$ of 49 / $51 \mathrm{v} / \mathrm{v}$ containing $0.1 \mathrm{~mol} / \mathrm{L} \mathrm{NaSCN}$; this warrants both density matching of the suspending medium and the particles and screening of the particle charges.

To determine the radius of the PS-PNiPAM particles, we perform static and dynamic light scattering experiments using very dilute dispersions. The static data are fitted with the Guinier approximation to obtain the radius of gyration $R_{g}$, which is then converted to a hard sphere radius $R=R_{g} / \sqrt{3 / 5}$ [35]. For the analysis of the dynamic light scattering data, we use the Stokes-Einstein relation to obtain the hydrodynamic radius $R_{h}$. Both techniques yield identical results, $R=R_{h}=117 \pm 3 \mathrm{~nm}$. Because the PNiPAM shell is very small, a determination of its size is not possible using light scattering techniques. However, the range of the steric repulsion due to the PNiPAM shell is sufficiently shortranged that the aggregation behavior of our particles depends on temperature owing to the temperature dependent dimensions of PNiPAM [36]. The system under investigation, a suspension with a particle volume fraction of $\phi=0.185$, remains dispersed up to a temperature of $T \approx 18^{\circ} \mathrm{C}$. Beyond this temperature aggregation sets in. Thus, in contrast to other temperature sensitive colloidal systems [30,34,37-39], aggregation is here not induced by the phase transition of the grafted polymer layer itself. Our system aggregates at temperatures far below the lower critical solution temperature of PNiPAM, which is at $\approx 32^{\circ} \mathrm{C}$. This clearly denotes that the origin of the temperature induced attraction is set by the temperature dependent range of the steric repulsion; at elevated temperatures, the repulsion becomes too short-ranged to effectively screen the Van der Waals attractions between the PS cores, which results in aggregation and gelation. In this work, we study the aggregation and gelation at $27^{\circ} \mathrm{C}$. At this temperature, we estimate the magnitude of the attraction to be of the order of $50 \mathrm{kT}$. This rough estimate is based on the volume fraction dependence of the shear modulus, which we can describe by a power law, $G=\left(\kappa_{o} / R\right) \phi^{\alpha}$ with $\kappa_{\mathrm{o}}$ a spring constant accounting for the resistance to stretching a bond [40]; for our gel we find $\alpha=4.1 \pm 0.1$ and $\kappa_{\mathrm{o}} \approx 1.9 \mathrm{~N} / \mathrm{m}$. We assume Van der Waals (VdW) interactions between PS spheres in water and estimate the magnitude of the attraction by taking the second derivative of the $\mathrm{VdW}$ potential to match the experimental value of $\kappa_{\mathrm{o}}$. In our experiments, the magnitude of the attraction is rather large, such that we expect that gelation is mainly governed by diffusion limited aggregation. This is also consistent with the power-law dependence of the shear modulus, which agrees with that typically found for fractal gels [41-43].

\section{B. Rheology}

Our rheological experiments are performed using a commercial rheometer (Anton Paar MCR502) equipped with a stainless steel cone and plate (cone radius $12.5 \mathrm{~mm}$; cone angle $1^{\circ}$ ). Experiments performed with a larger cone show some quantitative differences that are, however, always proportional to the results obtained with the smaller cone. Tests with roughened tools (surface roughness $\approx 50 \mu \mathrm{m}$ ) reveal that wall slip is irrelevant in our experiments. To minimize evaporation we use a solvent trap. The temperature within the gap is precisely controlled by a Peltier stage and Peltier hood. To account for the delayed heat transfer to the sample upon a change of the temperature of stage and hood, we performed a number of calibration experiments, concluding that temperature ramps with $\mathrm{d} T / \mathrm{d} t \leq 2.4{ }^{\circ} \mathrm{C} / \mathrm{min}$ are slow enough for the sample temperature to match that of the Peltier elements monitored in our instrument. To keep our temperature ramp as short as possible, while ensuring a homogeneous temperature within the gap at all times, we thus chose the heating rate of $2.4{ }^{\circ} \mathrm{C} / \mathrm{min}$ for our experiments. Repeated oscillatory strain experiments probing the effect of a temperature ramp from 15 to $27^{\circ} \mathrm{C}$ on the mechanical properties of our system in the linear range reveal that our system exhibits an aggregation and gelation behavior that is reproducible 
within 5\%. This reproducibility guarantees that experiments performed with fresh sample loads yield consistent results. All rheological experiments reported in this work are performed by subjecting fresh samples to the aforementioned temperature ramp, followed by the desired time dependent rheological test.

\section{Diffusing wave spectroscopy}

Additional experiments exploring the evolution of the dynamics of our system upon a temperature quench from 15 to $27^{\circ} \mathrm{C}$ are performed with a home built DWS setup, designed to perform transmission experiments over a wide range of lag times $\tau$. To control the temperature, we use a temperature cell equipped with two parallel glass windows that allow for optical access. This cell is filled with filtered water and the temperature is controlled to a precision of $0.1^{\circ} \mathrm{C}$ via a thermostat that is connected to a water circuit located within the walls of the temperature cell. The temperature quenches are performed by placing the samples equilibrated beforehand at $15^{\circ} \mathrm{C}$ into the temperature cell that has been equilibrated to $27^{\circ} \mathrm{C}$. The heating rate within the sample cell is thus set by the heat transfer rate. We perform experiments using sample cells with two different path lengths, $L=1 \mathrm{~mm}$ and $L=2 \mathrm{~mm}$, where we find that the set temperature of $27^{\circ} \mathrm{C}$ is reached within 100 and $200 \mathrm{~s}$, respectively. The transport mean free path of our gel is $l^{*}=14.4 \mu \mathrm{m}$, such that the condition for strong multiple scattering $L / l^{*}>10$ is fulfilled for both sample cells [44]. The use of two different optical path-lengths is justified by the wide range of time scales we aim to assess. Indeed, for the diffusive photon transport exploited in DWS the optical path length defines the number of independent scattering steps [44]. For systems with complex dynamics exhibiting correlation functions with more than one decay, the number of independent scattering steps determines the amplitude of each decay. Processes involving small displacements, such as thermal gel fluctuations, will lead to a larger decay of the correlation function when we use a cell with a large path length, since the added displacements along the scattering path will be larger; this facilitates the analysis of such fluctuations. However, when the amplitude of the decay due to such fast dynamical process is large, the contributions of slower processes are masked. By using a smaller path length, the contributions of fast dynamics are decreased, which enables us to assess the long time processes more accurately.

The optical design of the DWS setup is similar to that reported in [45]. As a light source, we use a solid-state laser with a wavelength of $\lambda=532 \mathrm{~nm}$. The beam is expanded to $8 \mathrm{~mm}$ and impinges the sample cell at normal incidence. The transmitted light is collected by a lens and split into perpendicular directions. In one of the directions the temporal evolution of the far field speckle pattern is recorded with a Pulnix CCD-camera using an exposure time of $2 \mathrm{~ms}$. These images are used to calculate the time resolved correlation signals $c_{I}(t, \tau)=\left\langle I_{p}(t) \cdot I_{p}(t+\tau)\right\rangle_{p} /\left(\left\langle I_{p}(t)\right\rangle_{p} \cdot\left\langle I_{p}(t+\tau)\right\rangle_{p}\right)-1$, where $I_{p}(t)$ is the scattered intensity at pixel $p$ and time $t$ $[46,47]$. Using these signals, we select time windows during which the dynamics can be considered as almost stationary to form time averages, recovering thereby the familiar intensity correlation function $g_{2}(\tau)-1=\overline{c_{I}(t, \tau)}$ classically determined in dynamic light scattering experiments, $\ldots$ denoting the time average. In the second detection line, we use a single speckle detection scheme to determine the short-time behavior of $g_{2}(\tau)-1$. To ensure ensemble averaging, we place a slowly rotating ground glass in front of the detector $[45,48]$. The light is collected by a single mode fiber, split in two and detected by a couple of avalanche detectors. The signals are cross correlated to determine $g_{2}(\tau)-1$

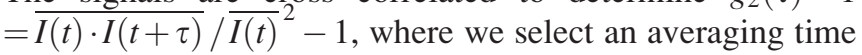
that guarantees that the dynamics of the evolving gel is not significantly changing during the acquisition of a given correlation function.

\section{RESULTS AND DISCUSSION}

\section{A. Gel formation, coarsening, and aging of the quiescent system}

The main advantage of using colloids with temperature dependent attractions is that aggregation can be induced by a temperature quench, thereby avoiding the use of a somewhat ill-defined shear rejuvenation procedure to control the history of the sample. In this work, we control the history of the gel by quenching a suspension of PS-PNiPAM particles with $\phi=0.185$ from 15 to $27^{\circ} \mathrm{C}$. At $15^{\circ} \mathrm{C}$ the particles are fully dispersed while they interact with an attraction strength of $\approx 50 \mathrm{kT}$ at $27^{\circ} \mathrm{C}$. The start of the quench is defined as $t=0$. At this moment, the temperature within the sample starts to increase. This results in an increase of the attraction strength which eventually induces aggregation. Because the quench is not instantaneous the kinetics of aggregation at the beginning of our experiments is somewhat ill-defined, as we effectively ramp through the attraction strength of $\approx 1$ to $\approx 50 \mathrm{kT}$ during the temperature quench. However, tests probing the aging properties of gels obtained by increasing the temperature at different rates reveal that at sufficiently long aging times the gel properties are independent of the quench rate. Our investigations are restricted to long aging times, where we can neglect the details of the temperature history experienced during the temperature quench.

\section{Dynamical characteristics}

The effect of the rise in attraction strength with increasing the temperature is readily observed in DWS, where we reach the final set temperature of $27^{\circ} \mathrm{C}$ within $100 \mathrm{~s}$ using sample cells with an optical path length of $L=1 \mathrm{~mm}$. As evidenced by the time dependence of the intensity correlation functions shown in Fig. 1(a), the dynamics of the system exhibits a strong temporal evolution as a response to the temperature quench. Surprisingly, the correlation functions develop a three-step decay. The first decay observed at short lag times $\tau$ is related to fluctuations of network strands similar to those observed in strongly aggregated fractal gels [49]. The magnitude of this decay as well as the cross-over time to an intermediate plateau value decrease with sample age, reflecting an increase in the gel elasticity. In the long-time limit of the correlation function, the third decay shifts systematically to 


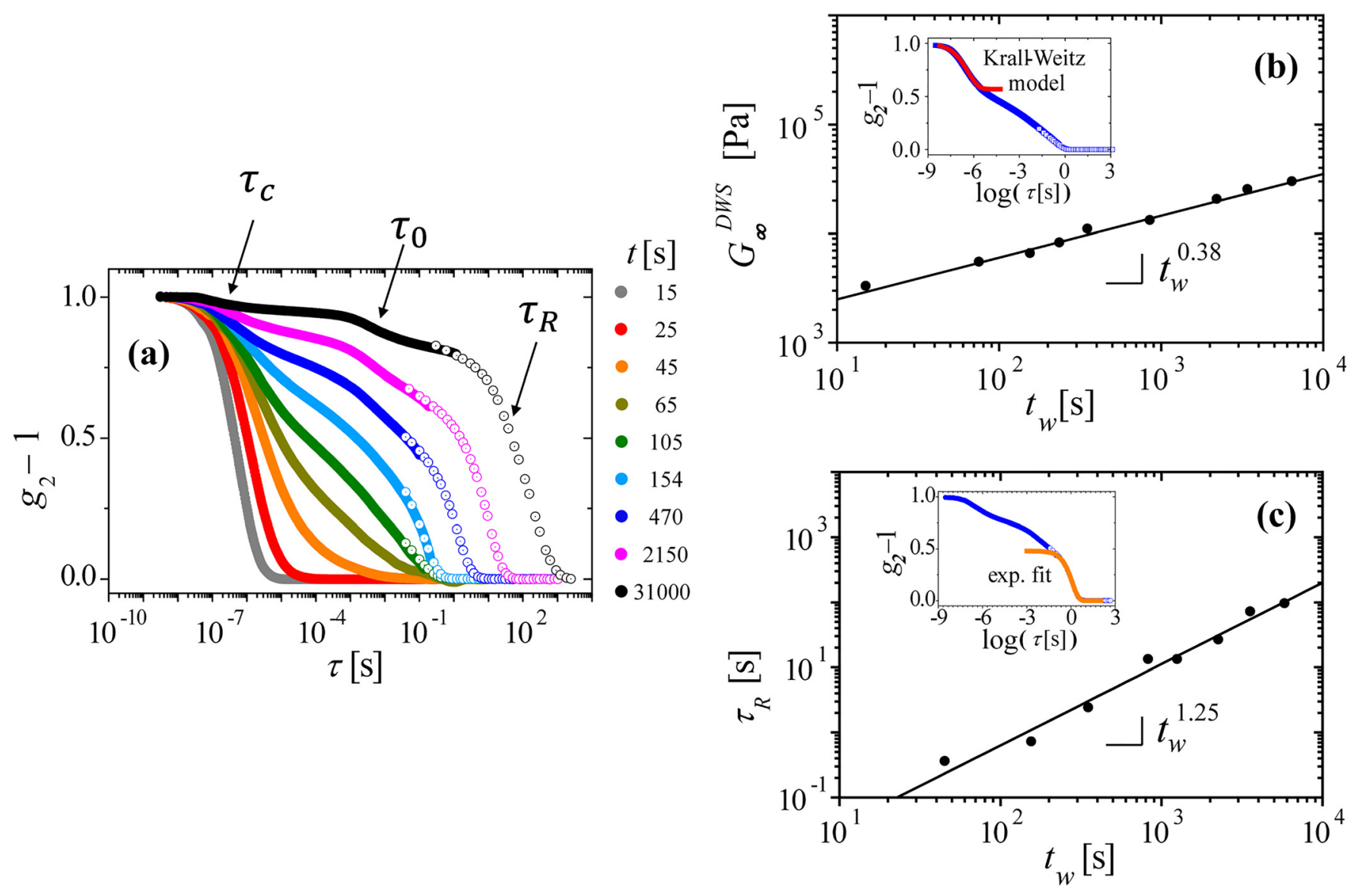

FIG. 1. Evolution of dynamical properties of thermosensitive PS-PNiPAM system in response to a temperature quench from $T=15^{\circ} \mathrm{C}$ to $T=27^{\circ} \mathrm{C}$. (a) Intensity correlation functions $g_{2}-1$ obtained with an optical path length of $L=1 \mathrm{~mm}$. The time $t$ indicated in the legends corresponds to the time interval between the start of the temperature quench and the midpoint of the time averaged measurement of $g_{2}-1$. Filled and open symbols refer to, respectively, singlespeckle and multispeckle acquisitions. (b) High frequency elastic modulus $G_{\infty}^{D W S}$ as a function of the gel age $t_{w}=t-t_{g}$, with $t_{g}=100 \mathrm{~s}$ the gelation time. The moduli are obtained by fitting the short-time decay of $g_{2}-1$ obtained with $L=2 \mathrm{~mm}$ with the Krall-Weitz model [21,26] and converting $\tau_{c}$ into $G_{\infty}^{D W S}$ (see text). The age dependence of $G_{\infty}^{D W S}$ is best described by the power law indicated as solid line, $G_{\infty}^{D W S} \propto t_{w}{ }^{0.38 \pm 0.05}$. Inset: Example of fit for the data obtained at $t=470 \mathrm{~s}$. (c) Age dependence of the structural relaxation time $\tau_{R}$. The relaxation time is obtained by approximating the long-time limit of $g_{2}-1$ obtained with $L=1 \mathrm{~mm}$ by a single exponential function. The age dependence of $\tau_{R}$ is best described by the power law indicated as a solid line, $\tau_{R} \propto t_{w}{ }^{1.25} \pm 0.05$. Inset: Example of fit for the data obtained at $t=470 \mathrm{~s}$.

longer times as the system ages. This decay is due to residual structural relaxation processes typical of aging systems [50]. The truly unusual feature is the intermediate, second, decay in the correlation functions. The characteristic decay time is here age-independent, while the magnitude of the decay systematically decreases with increasing sample age.

A quantitative description of this decay is beyond the scope of this paper. Qualitatively, we can understand this decay as being due to the diffusion of a small number of single particles or small clusters that are not yet part of the network. To appreciate this let us recall that light passing through a turbid medium will be scattered multiple times before exiting the sample. We generally treat this problem as a photon diffusion problem, describing the photon path through the sample as a random walk with step length $l^{*}$ [44]. For a system solely composed of freely diffusing particles, the intensity correlation function obtained in transmission is reasonably described by an exponential decay with a characteristic time that sensitively depends on the number of independent scattering steps, $\left(L / l^{*}\right)^{2}$, encountered while the light passes the sample of thickness $L$. As the added displacements of the scattering sites (the particles) along a scattering path will contribute to the phase shift that leads to the decay of the intensity correlation function, we expect that the decay time is $\tau_{o}{ }^{*} \approx 1 /\left(2 \cdot k_{o}{ }^{2} \cdot\left(L / l^{*}\right)^{2} \cdot D_{o}\right)$, with $D_{o}$ the free diffusion coefficient of the particle and $k_{o}=2 \cdot \pi \cdot n / \lambda$ the wave vector, $n$ the refractive index of the suspending medium, and $\lambda$ the wavelength of the incident light. In contrast, if we consider that at most one particle along the scattering path is free to move, while all others are not, the motion of only that particle will lead to a phase shift. The decay time characterizing the partial dephasing due to the motion of this particle will be independent of the number of independent scattering steps, $\tau_{o} \approx 1 /\left(2 \cdot k_{o}^{2} \cdot D_{o}\right)$, and thus significantly slower than that expected if all particles of the system were free to diffuse. Calculating this time for our PSPNiPAM particles we find $\tau_{o}=1 \mathrm{~ms}$ in reasonable agreement with the time scale at which we observe the second decay. This strongly suggests that this decay is indeed due to the motion of a small fraction of single particles that are not yet part of the network. The decreasing magnitude of the intermediate decay with sample age indicates that the number of these mobile particles is a decreasing function of the age of the sample $[51,52]$. We can thus conclude that the temporal 
evolution of our gel involves the incorporation of mobile particles into the arrested network structure.

Such incorporation has recently been suggested to lead to an increase of the high frequency elasticity $G_{\infty}$ [5]. To get a the first quantitative assessment of this increase, we model the first decay in $g_{2}-1$ using the model developed by Krall and Weitz for the internal fluctuations of fractal gels [49,53]. Let us note, however, that the particle volume fraction of our system is somewhat too high to fully justify this approach. Within the Krall-Weitz model, the constraint internal fluctuations of gels can be modeled as $\left\langle\Delta r^{2}\right\rangle=\delta^{2}\left(1-\exp \left\{-\tau / \tau_{c}\right\}^{p}\right)$ with $\left\langle\Delta r^{2}\right\rangle$ the mean square displacement, $\delta^{2}$ the maximum mean square displacement of gel strands, $p \approx 0.7$, and $\tau_{c}$ a characteristic time that relates to the elastic modulus as $G_{\infty}^{D W S}$ $=6 \pi \eta /\left(\tau_{c} / 0.35\right)$. Using the approximation for DWS data, $g_{2}-1 \approx \exp \left\{-1 / 3\left\langle\Delta r^{2}\right\rangle k_{o}^{2}\left(L / l^{*}\right)^{2}\right\}$, this model describes the initial decay of our correlation functions reasonably well. As an example, we show in the inset of Fig. 1(b) the fit obtained for the data acquired at $t=470 \mathrm{~s}$ with a sample cell with $L=2 \mathrm{~mm}$. Since the amplitude of the first decay is larger in the correlation functions obtained with $L=2 \mathrm{~mm}$ than in those obtained with $L=1 \mathrm{~mm}$ [for comparison see the inset of Fig. 1(c)], we use the data acquired with $L=2 \mathrm{~mm}$ for the systematic analysis of the first decay, converting $\tau_{c}$ into $G_{\infty}^{D W S}$. The results obtained are shown in the main graph of Fig. 1(b), where $t_{w}$ denotes the age of the gel defined as $t-t_{g}$ with $t_{g}$ the gelation time, which we estimate to be $\approx 100 \mathrm{~s}$ in our DWS experiments. The age dependence of $G_{\infty}^{D W S}$ is best described by a weak power law, $G_{\infty}^{D W S} \propto t_{w}{ }^{0.38 \pm 0.05}$, broadly consistent with the results obtained for other colloidal gels [37,54-56].

For the characterization of the long-time relaxation $\tau_{R}$, we use the data obtained with $L=1 \mathrm{~mm}$, where the amplitude of the long-time decay is larger than in the data obtained with $L=2 \mathrm{~mm}$. The long-time relaxation is reasonably well described by a single exponential decay, as shown for $t=470 \mathrm{~s}$ in the inset of Fig. 1(c). Remarkably, we find that $\tau_{R} \propto t_{w}{ }^{1.25 \pm 0.05}$. Such dependence is somewhat unusual as the structural relaxation time is generally found to depend linearly or sublinearly on $t_{w}$ [21]. An aging exponent larger than one in principle indicates that the relaxation time will at some point exceed the sample age. Such unusual aging behavior has been reported previously for very strongly aggregated gels, where it was shown that aging eventually leads to a catastrophic breakdown of the gel rather than a vanishing long-time relaxation [57]. Hyperaging has also been observed in simulations of attractive glasses, where it was denoted that, while the structural relaxation grows faster than the aging time, the bond-life time grows slower than the aging time [58]. Such decoupling of the evolution of bond lifetime and structural relaxation indicates that structural relaxations are not or only partly linked to the bond-life time. In fact, structural relaxations in strong gels have been shown to be governed by intermittent events involving rearrangements on larger length scales [59]. The structural relaxation process of our gel displays an almost single exponential behavior, which is consistent with a scenario where intermittent rearrangements occurring randomly in space and time are at the origin of structural relaxation [60].
Indeed, if we consider that any scattering path crossing a zone that has been restructured by an intermittent event will be fully dephased, the field correlation function $g_{l}$ will be a measure of the fraction of the paths that has not yet been dephased. For randomly occurring events, one can then write: $=\sqrt{g_{2}(\tau)-1} \approx \exp \left\{-\Gamma_{e} \cdot\left(V_{e} / V_{s}\right) \cdot\left(L / l^{*}\right)^{2} \cdot \tau\right\}$, with $\Gamma_{e}$ the rate of restructuring events occurring within the scattering volume of size $V_{s}$ and $V_{e}$ the typical volume of the zone rearranged during an intermittent restructuring event [60]. In analogy we would expect that for dynamic light scattering experiments in the single scattering limit $g_{1}(\tau)=\exp \left\{-\Gamma_{e} \cdot\left(V_{e} / V_{s}\right) \cdot \tau\right\}$, provided that the displacement of the scattering sites within the rearranged volume is large compared to $1 / q$, with $q$ the scattering vector. A number of gel studies $[37,59,61-63]$ report instead correlation functions that are best described by a compressed exponential, displaying relaxation times that depend on $1 / q$ reminiscent of ballistic-like motion. As discussed in [59], these characteristics are still consistent with intermittent dynamics; they indicate that for the experimentally accessible $q$-range the displacements of the scattering sites within the restructured zone are insufficient to fully dephase the scattering contributions of these sites. We then recover $q$-dependence and we expect compressed exponentials when the direction in the displacement of the scattering sites is maintained in subsequent restructuring events. In contrast, our experiments performed in the multiple scattering limit can be regarded as a high $q$ experiment, where we recover a simple exponential relaxation, which is entirely set by the restructuring rate [59].

\section{Linear mechanical characteristics}

To assess the evolving characteristics of our gel in rheology, we first perform a series of oscillatory shear experiments. The temperature history of the gel is here controlled by imposing a heating rate of $2.4^{\circ} \mathrm{C} / \mathrm{min}$ increasing the temperature from 15 to $27^{\circ} \mathrm{C}$. We monitor the evolution of the system during and after the quench by measuring the storage $G^{\prime}$ and loss modulus $G^{\prime \prime}$ within the linear range $\left(\gamma=10^{-3}\right)$ at an angular frequency of $\omega=10 \mathrm{rad} / \mathrm{s}$, well below the time scales defining Brownian dynamics, the Brownian diffusion time of our particles being $\tau_{B}=7 \mathrm{~ms}$. The moment we start the temperature quench is defined as $t=0$. As the temperature increases, the system becomes unstable and the particles aggregate, which rapidly results in the formation of a stressbearing network, as evidenced by the development of the storage and loss moduli shown in Fig. 2(a). During the initial increase in temperature, the moduli are too low to be resolved, the loss modulus $G^{\prime \prime}$ initially dominating over $G^{\prime}$. At $t \approx 200$ s $G^{\prime}$ becomes the dominant modulus; both moduli then rapidly rise by several orders of magnitude as the temperature reaches $27^{\circ} \mathrm{C}$. We identify the gel point as the moment at which $G^{\prime \prime}=G^{\prime}$ and define this time as $t_{w}=0$. In the long time limit of our experiment, the gel elasticity is of the order of $10^{4} \mathrm{~Pa}$ comparable to the moduli reported for the thermo-reversible gels studied by Gopalakrishnan and Zukoski [30]. 

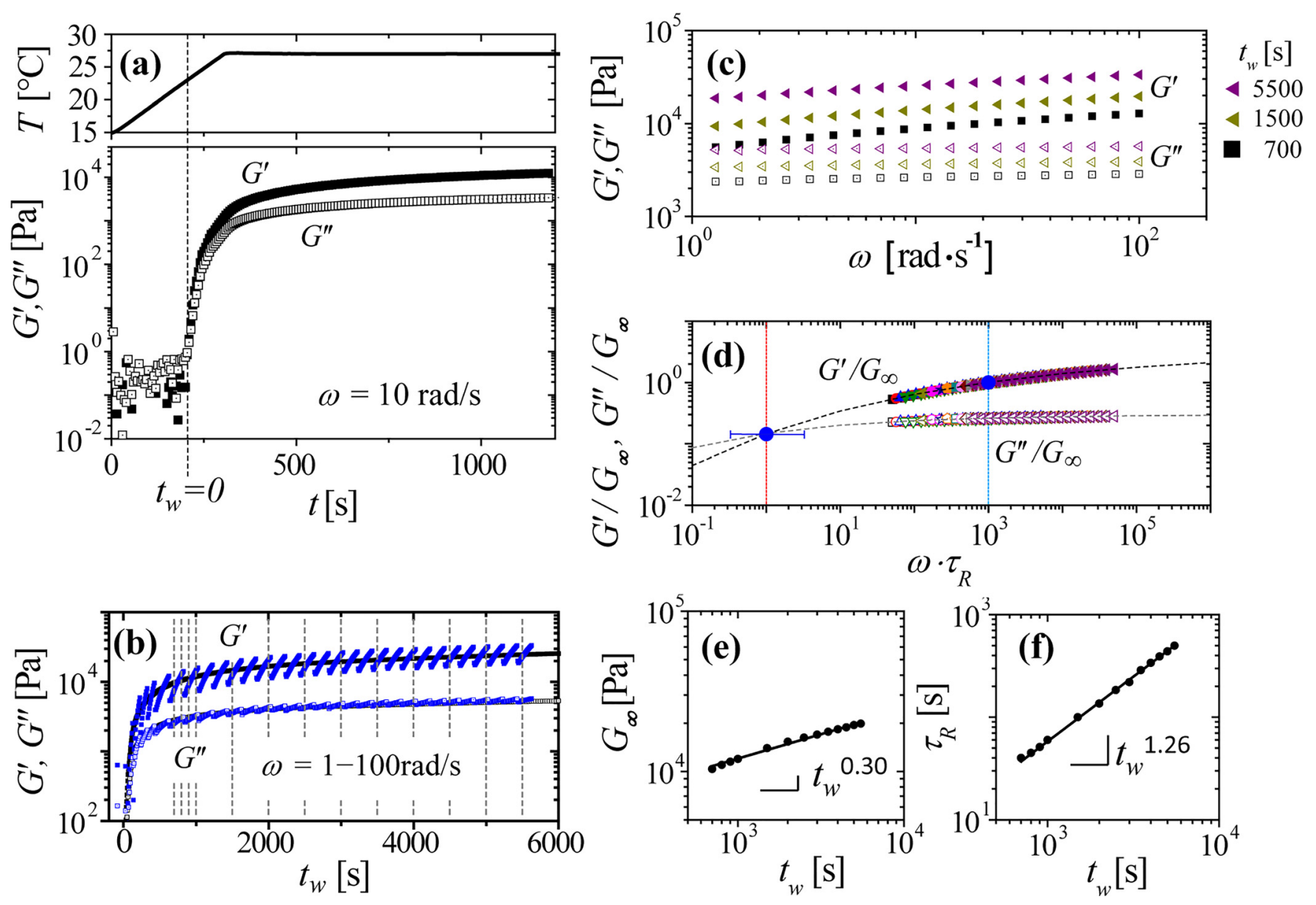

FIG. 2. Evolution of rheological properties of PS-PNiPAM system in response to a temperature quench from $T=15^{\circ} \mathrm{C}$ to $T=27^{\circ} \mathrm{C}$. (a) Time dependence of $G^{\prime}$ and $G^{\prime \prime}$ measured at an angular frequency of $\omega=10 \mathrm{rad} / \mathrm{s}$ and a strain of $\gamma=10^{-3}$. The age of the gel $t_{w}$ is defined relative to the gel point denoted as dashed vertical line. (b) The black symbols denote the data shown in (a), the blue symbols correspond to data obtained by repeatedly ramping the frequency from 1 to $100 \mathrm{rad} / \mathrm{s}$. Quasi-instantaneous frequency spectra are reconstructed by interpolation at the gel ages indicated by the dashed vertical lines. Three examples of these frequency sweeps are shown in (c). (d) Quasi-instantaneous frequency spectra scaled to obtain a unique master curve; this requires a normalization of both the frequency and the moduli. To estimate the cross-over frequency $\omega_{R}=1 / \tau_{\mathrm{R}}$ at which $G^{\prime}=G^{\prime \prime}$ the master curves are fitted with exponential functions. For the estimation of the absolute magnitude of $G_{\infty}$, we use $G^{\prime}$ at a scaled frequency of $\omega \cdot \tau_{R}=10^{3}$ indicated by a vertical line. (e) Age dependence of $G_{\infty}$. (f) Age dependence of $\tau_{R}$. The power laws describing $G_{\infty}$ and $\tau_{R}, G_{\infty} \propto t_{w}{ }^{0.30 \pm 0.03}$ and $\tau_{R} \propto t_{w}{ }^{1.26 \pm 0.02}$, are in agreement with the DWS results.

To obtain measures of the evolving characteristics of the gel similar to those obtained in DWS experiments, we perform a second type of measurement. Instead of monitoring the temporal development of the moduli at a fixed frequency, we continuously vary the probe frequency from 1 to $100 \mathrm{rad} / \mathrm{s}$ during the evolution of the gel, as shown in Fig. 2(b). Let us note that we carefully checked that the small mechanical perturbations generated by applying an oscillatory strain of $\gamma=10^{-3}$ during the structural evolution of the gel do not affect the gel structure significantly; any experiment performed at a given $t_{w}$ yields identical results independently of whether we applied an oscillatory strain during gelation or not. Our frequency dependent experiments permit for the determination of the frequency dependence of $G^{\prime}$ and $G^{\prime \prime}$ at different ages. Indeed, each data point in Fig. 2(b) is obtained by averaging the signal over $10 \mathrm{~s}$. Using interpolation schemes, we can thus reconstruct quasi-instantaneous frequency spectra. Three examples of such spectra are shown in Fig. 2(c); within the limited frequency range explored $G^{\prime}$ and $G^{\prime \prime}$ display a more pronounced frequency dependence for the young gel than for the older gels. These spectra can be scaled onto a single master curve by normalizing both the frequency and the moduli. This is shown in Fig. 2(d), where we demonstrate the scaling for the series of spectra obtained at the ages denoted by the dotted lines in Fig. 2(b). Such scaling behavior indicates that the frequency dependence of $G^{\prime}$ and $G^{\prime \prime}$ remains essentially the same during the gel evolution, merely exhibiting age-dependent scaling parameters. Based on our results obtained in DWS, we can expect that these parameters relate to $\tau_{R}$ and $G_{\infty}$. To obtain an approximate gauge of the absolute magnitude of $\tau_{R}$ we use the master curve and extrapolate the data using exponential fits to determine the frequency $\omega_{R}$ at which $G^{\prime}=G^{\prime \prime}$, as shown in Fig. 2(d). Using the scaling parameters used to produce the master-curve we then recover $\tau_{R}=1 / \omega_{R}$ as a function of $t_{w}$. According to this analysis, the structural relaxation times of the gel are more than a factor of $10^{4}$ larger than the Brownian diffusion time. They exhibit a power-law increase with increasing sample age, as shown in Fig. 2(f). Consistent with the DWS results we find that $\tau_{R} \propto t_{w}{ }^{1.26 \pm 0.02}$. To get an estimate for the magnitude of the high frequency modulus $G_{\infty}$ we use $G^{\prime}$ at a scaled frequency of $\omega \cdot \tau_{R}=10^{3}$. As shown in 
Fig. 2(e), the dependence of $G_{\infty}$ on $t_{w}$ can be described as $G_{\infty} \propto t_{w}{ }^{0.30 \pm 0.03}$, a power law that is within error bars consistent with that obtained from the DWS data shown in Fig. 1(c). Let us here stress that for the considerations of the agedependent viscosity discussed in Sec. III B, the power-law dependences of $\tau_{R}$ and $G_{\infty}$ on $t_{w}$ are highly relevant, while the exact magnitude of either quantity is less important. Even though our choice of how to gauge the absolute magnitude of $\tau_{R}$ and $G_{\infty}$ is rather arbitrary, the scaling of the data shown in Fig. 2(d) ensures an accuracy of $\sim 5 \%$ for the relative values of $\tau_{R}$ and $G_{\infty}$. The dependences of $\tau_{R}$ and $G_{\infty}$ on $t_{w}$ are thus determined with relatively high accuracy.

In summary, both DWS and oscillatory shear experiments indicate that the temporal evolution of the gel leads to an increase of the elasticity and the structural relaxation time, both parameters exhibiting a power-law scaling with $t_{w}$. Moreover, the results obtained in DWS indicate that the gel evolution involves a decrease in the fraction of mobile particles, and it is likely that this change is associated with the development of a coarser gel structure, as observed in a number of weakly aggregated gels $[5,63,64]$. Thus, one could conceive that the evolution of the elasticity and the structural relaxation time of our gel would be entirely due to coarsening, a process that we can consider as corresponding to a delayed continuation of the initial aggregation process. However, with respect to structural relaxations our gels exhibit hyper-aging in agreement with simulations of attractive glasses. For these glasses, it was found that the evolution of the bond-life time does not directly relate to the age dependence of the structural relaxations [29]. Considering the mobility of our particles as a measure of the bond-life time, we can therefore presume that the evolution of the structural relaxation with the sample age does not directly relate to coarsening.

\section{B. Evolving gel under stress}

\section{Creep behavior}

To elucidate how aging and/or coarsening impact the creep behavior of our gel, we perform series of creep experiments. In a first series, we explore the response of the gel to applied stresses of varying magnitude starting the experiment at $t_{w}=1000 \mathrm{~s}$, an age beyond the regime where the gel exhibits significant hardening in time [see Fig. 2(b)]. At low enough stresses, the application of a constant stress results in an instantaneous elastic response, which is somewhat masked by ringing due to the rheometer inertia $[65,66]$. This initial response is followed by an increase in strain at a continuously decreasing strain rate, as shown in Fig. 3, where we display the lag-time dependence of the strain $\gamma$, the compliance $J$ and the strain rate $\dot{\gamma}$ as a result of applying different stresses $\sigma$. Interestingly, the stress-dependent response functions of our gels can be divided in three regimes. Below $\sigma_{c} \approx 50 \mathrm{~Pa}$, the lag-time dependence of $J(\tau)$ is essentially independent of the stress applied. Just above $\sigma_{c}$ the initial instantaneous response to a stress can still be described by a unique function, but deviations appear at longer lag times; the larger the stress the larger $J(\tau)$. Increasing the stress beyond $\sigma_{y} \approx 100 \mathrm{~Pa}$, the system exhibits delayed yielding within the experimental time window. Consistent with the yield behavior of other colloidal gels, we find that the
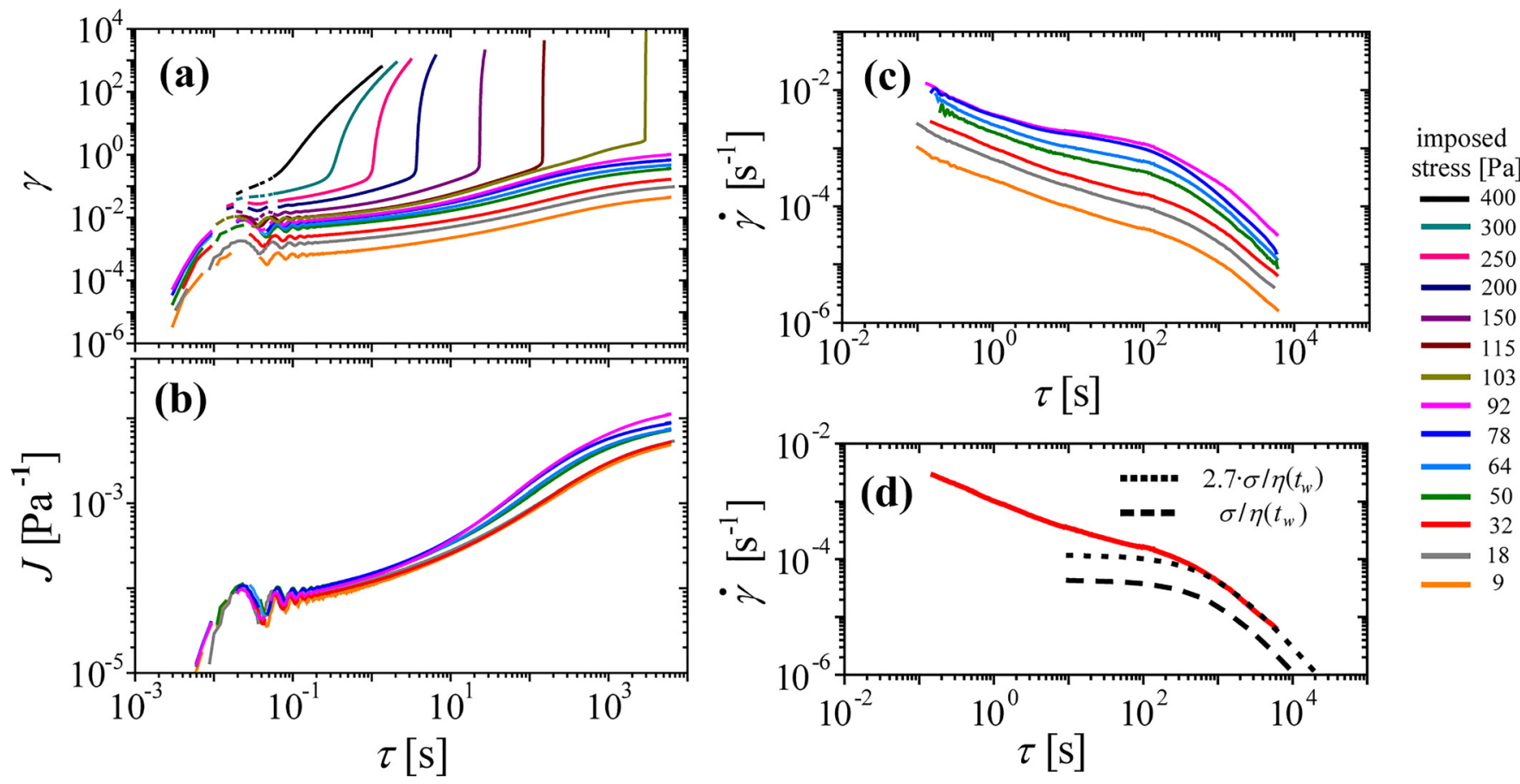

FIG. 3. Creep response of PS-PNiPAM gel starting stress application at $t_{w}=1000 \mathrm{~s}$. (a) Lag-time dependence of the strain obtained for different applied stresses. Beyond $92 \mathrm{~Pa}$ delayed yielding is observed within the limited lag-time window of our experiment. (b) Lag-time dependence of the compliance for stresses up to $92 \mathrm{~Pa}$. Beyond $32 \mathrm{~Pa}$ the long-time limit of $J$ is stress dependent. (c) Lag-time dependence of the strain rate. (d) Comparison between the experimental strain rates measured with $\sigma=32 \mathrm{~Pa}$ and the strain rates expected from the age-dependent viscosity $\eta\left(t_{w}\right)=G_{\infty}\left(t_{w}\right) \cdot \tau_{R}\left(t_{w}\right)$. Qualitative agreement in the functional development of the experimental data and the expectation values is only obtained in the long time limit of the experiment. For better comparison, the expectation values multiplied by a factor of 2.7 are shown as a black dotted line. 
yielding time is a strong function of the stress. For our gels, the stress-dependence of the yielding times resembles that reported in [30]; it is best described by a power law reminiscent of the behavior reported for carbopol [67]. Delayed yielding in gels is generally attributed to a competition of bond breakage and formation, or alternatively to a competition between strand break-up and reformation, where yield is obtained when the connectivity of the system cannot be maintained $[26,30,32,33,68]$. In contrast, recent simulations show that gels maintain their integrity when subjected to stresses that only marginally exceed the yield stress and that yield involves a loss of only a tiny fraction of bonds [31]. The focus of this paper is on the precursor of such delayed failure, i.e., the creep responses and the impact of aging on these response functions.

To a first approximation, we can assume that the strain rate dependence on the lag time $\tau$ will be governed by the age-dependent viscosity $\eta\left(t_{w}\right)=G_{\infty}\left(t_{w}\right) \cdot \tau_{\mathrm{R}}\left(t_{w}\right) \propto t_{w}{ }^{1.55}$. Within the time interval of the creep experiments the viscosity of the system increases, such that we naturally expect the strain rate to be a decaying function of $\tau$. In Fig. 3(d), we show as an example the response function obtained with $\sigma=32 \mathrm{~Pa}$ (continuous orange line) and compare it to that expected from the age-dependent viscosity (dashed black line) using for $G_{\infty}\left(t_{w}\right)$ and $\tau_{\mathrm{R}}\left(t_{w}\right)$ the values reported in Figs. 2(e) and 2(f), respectively. Considering the large uncertainty in the absolute values of either of these quantities, it is certainly not surprising that the absolute values of the calculated shear rates do not exactly match the experimental values. Even a qualitative agreement between the expectation values and the experimental data is only obtained in the long-time limit of the creep experiment; for better comparison we show the vertically shifted expectation values as a dotted black line. This comparison clearly shows that the development of the shear rates at short times significantly deviates from that expected assuming that the temporal evolution of the shear rate is entirely determined by the age-dependent viscosity. We will show later that this deviation is due to an additional contribution not considered in the assumption that creep is exclusively governed by loss contributions.

\section{Deconvolution of creep contributions}

To fully assess the contributions to creep, we systematically investigate the recoverable strain along the creep curve. As an example, we show in Fig. 4(a) the creep-recovery curves obtained for a gel that has aged to $t_{w}=1000 \mathrm{~s}$ at the moment we start the creep experiment applying a stress of $32 \mathrm{~Pa}$ below $\sigma_{c}$. Clearly, creep involves significant losses. However, a closer inspection reveals that energy is also stored during creep. In Figs. 4(b) and 4(c), we compare the time dependence of the strain magnitude obtained in creep to that obtained during recovery, $\gamma_{\text {rec }}=\left(\gamma^{*}-\gamma\right)$ with $\gamma^{*}$ the strain reached at the end of the creep experiment. In both cases, we observe that the system exhibits an instantaneous strain response of the same order of magnitude. However, the recovered strain substantially exceeds the instantaneously stored/recovered strain, and it exhibits significant time dependence. This shows that creep not only leads to dissipative losses, but also allows for delayed elastic storage. Our recovery experiments enable us to deconvolute the contributions to creep in an instantaneous and delayed elastic strain, $\gamma_{I}$ and $\gamma_{D}$, and a lost strain $\gamma_{L}$, as shown in Fig. 4(d), where $\gamma_{T O T}=\gamma_{I}+\gamma_{D}+\gamma_{L}$. Both the delayed elastic strain and the lost strain are increasing functions of the time the stress is applied, while the instantaneous strain decays with increasing lag time.

\section{Stress hardening during creep}

To account for the decrease in $\gamma_{I}$ let us recall that the gel hardens in time. Presuming that this also impacts the elasticity of the gel under stress, we would expect the instantaneous strain recovered at a given lag time $\tau$ to be a decaying function of this additional time $\tau$ passed since the start of the creep experiment at $t_{w}=1000 \mathrm{~s}$. As denoted in Fig. 4(c), we determine $\gamma_{I}$ at $\tau=0.1 \mathrm{~s}$, at the end of the ringing process. To compare these values to those expected for the quiescent gel at a comparable time scale, we use the complex modulus $G^{*}$ measured at $\omega=10 \mathrm{rad} / \mathrm{s}$ and report as instantaneous strain $\gamma_{I}=\sigma / G_{0}^{*}$ in Fig. 4(d); the subscript zero denotes values obtained for the quiescent gel. The smaller strains observed in recovery suggest that the gel under stress hardens more than when left unperturbed. To verify this we perform a series of experiments monitoring the elasticity of the gel in oscillatory shear experiments after the system has fully recovered. Two examples of these experiments are shown in Fig. 5(a), where we compare the complex modulus $G^{*}$ $(\omega=10 \mathrm{rad} / \mathrm{s})$ of gels that have been subjected to a constant stress of, respectively, 32 and $78 \mathrm{~Pa}$ during the time period of $t_{w}=1000-1500 \mathrm{~s}$ to that of a quiescent gel which has been left unperturbed. The moduli obtained after creep and recovery experiments $G_{\sigma}^{*}$ are larger than those obtained for the quiescent gel $G_{0}^{*}$. Moreover, they are consistent with the elastic moduli $G_{\sigma}^{\#}$ determined as $\sigma / \gamma_{I}$, with $\gamma_{I}$ obtained in recovery. Clearly, the extent of hardening sensitively depends on the stress applied; the larger the stress the larger the stress-hardening effect.

However, imposing a stress over a given period of time is here not equivalent to temporarily increasing an "effective temperature" that would overall lead to faster aging. Indeed, in such case we would expect that both the high frequency modulus and the structural relaxation time would have been increased by the temporary stress application. This is, however, not the case, as shown in Fig. 5(b). In this graph, we compare the frequency dependent spectra of $G^{\prime}$ and $G^{\prime \prime}$ for gels of the same age, $t_{w}=4000 \mathrm{~s}$, having experienced the different stress histories displayed in Fig. 5(a). The magnitude of the moduli depends on the stress history, but the overall shape of the spectra remains the same. The moduli obtained for the gels subjected to the creep and recovery procedure can be scaled onto those of the unperturbed gel by applying a single normalization factor to the moduli, as shown in Fig. 5(c). This is in contrast to the scaling used to characterize the unperturbed gel, where we normalize both the moduli and the frequencies to obtain a unique master curve [see Fig. 2(d)]. It shows that the application of a stress induces hardening, but does not otherwise modify the relaxation process 

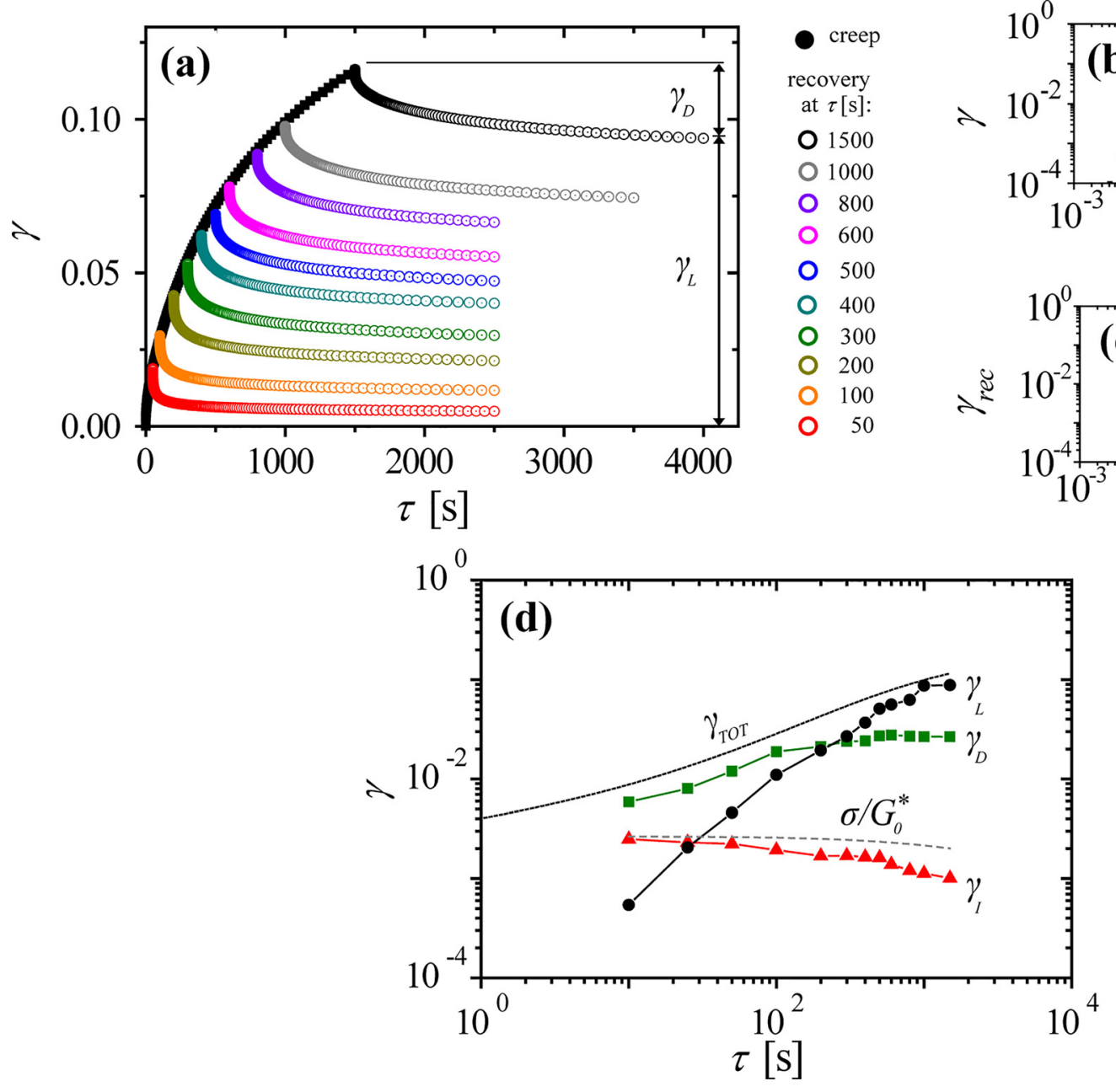

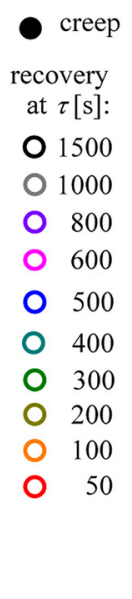

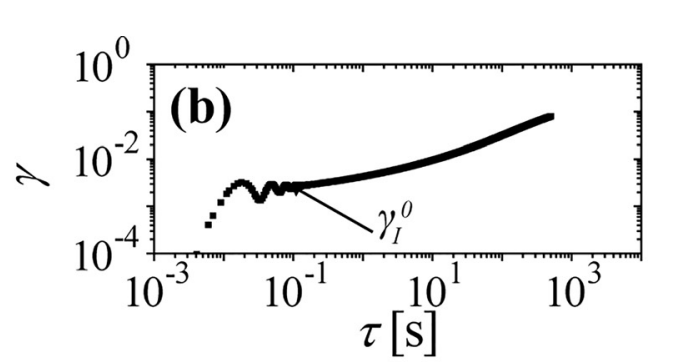

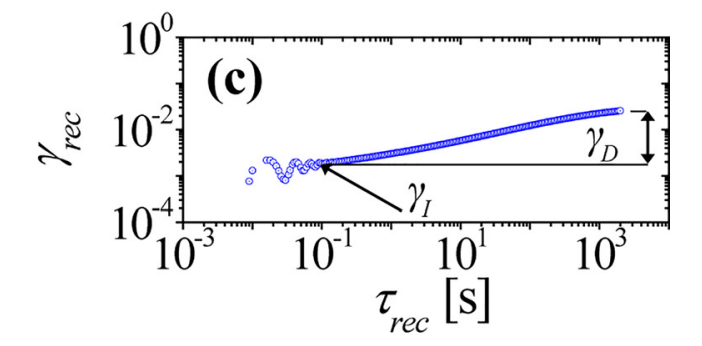

FIG. 4. (a) Systematic investigation of recoverable strain for creep experiment with $\sigma=32 \mathrm{~Pa}$, starting the stress application at $t_{w}=1000 \mathrm{~s}$. (b) Creep experiment in $\log -\log$ representation. (c) Recovery test started at $\tau=500 \mathrm{~s}$, where the recovered strain is defined as $\gamma_{\text {rec }}=\left(\gamma^{*}-\gamma\right)$ with $\gamma^{*}$ the maximum strain reached at the end of the creep experiment and $\tau_{r e c}=0$ at the moment the stress is set to zero. This experimental approach allows us to deconvolute the creep response into three distinct contributions: a lost strain $\gamma_{L}$, an instantaneous elastic strain $\gamma_{I}$, and a time-delayed elastic strain $\gamma_{D}$. (d) $\gamma_{I}$, $\gamma_{D}$, and $\gamma_{L}$ as a function of the time interval $\tau$ over which the stress was applied. The dotted line denotes the total strain $\gamma_{T O T}$ measured in the creep experiment. The dashed line corresponds to the expectation values of $\gamma_{I}$ based on the evolution of $G_{0}^{*}$ shown in Fig. 5(a).

of the system. This is also evidenced by the rate at which the elasticity increases after stress and recovery. Over the limited time window investigated this rate remains the same as that of the unperturbed gel; this is shown by the scaling behavior in Fig. 5(a), where the normalization of $G_{\sigma}^{*}$ with $f_{h}$ used for the normalization in Fig. 5(c) leads to an almost perfect collapse of the data on $G_{0}^{*}$. Such decoupling of the elasticity and the structural relaxation time indicates that the origin of the temporal evolution of either quantity is not the same.

To further explore the parameters impacting stress hardening, we investigate the relation of stress and time on the extent of hardening in more detail. For this we use the hardening factor $f_{h}=G_{\sigma}^{\#} / G_{0}^{*}$, where $G_{0}^{*}$ is the complex modulus of the unperturbed gel obtained at an age at which $G_{\sigma}^{\#}=\sigma / \gamma_{I}$ is measured. This quantity expresses the factorial increase of the elastic modulus due to stress application; it is equal to 1 when no stress is applied. The hardening factor depends on both the duration of stress application $\tau$ and the magnitude of the stress applied during creep $\sigma$, as shown in Fig. 6(a), where we display the reduced hardening factor $f_{h}-1$ obtained by applying various stresses at $t_{w}=1000 \mathrm{~s}$ and setting them back to zero at different lag times $\tau$. This quantity is directly proportional to $\sigma$, as evidenced by the scaling behavior shown in Fig. 6(b); a simple normalization of $f_{h}-1$ with the applied stress leads to a collapse of the different data sets obtained for $t_{w}=1000 \mathrm{~s} ; t_{w}$ denotes here the age of the sample at the start of the creep experiment and we will keep this notation in the following to refer to experiments started at different aging times. DWS experiments of the gel under stress (data not shown) reveal that the magnitude of the intermediate decay decreases more quickly for the gel under stress than for the quiescent gel. This indicates that stress hardening involves a loss of mobile particles, and we may envision that stretching the gel network exposes sites allowing for a more effective incorporation of mobile particles. This is consistent with results obtained in simulations of gels subjected to a continuous shear rate, where shear was found to favor bonding [69]. Because the stress hardening process leads to an increase of the elasticity, but not to a change of the relaxation time, we speculate that the age dependence of the elasticity relates to the mobility of particles, while structural relaxations involve 
stress imposed during creep [Pa] red: 32 blue: 78

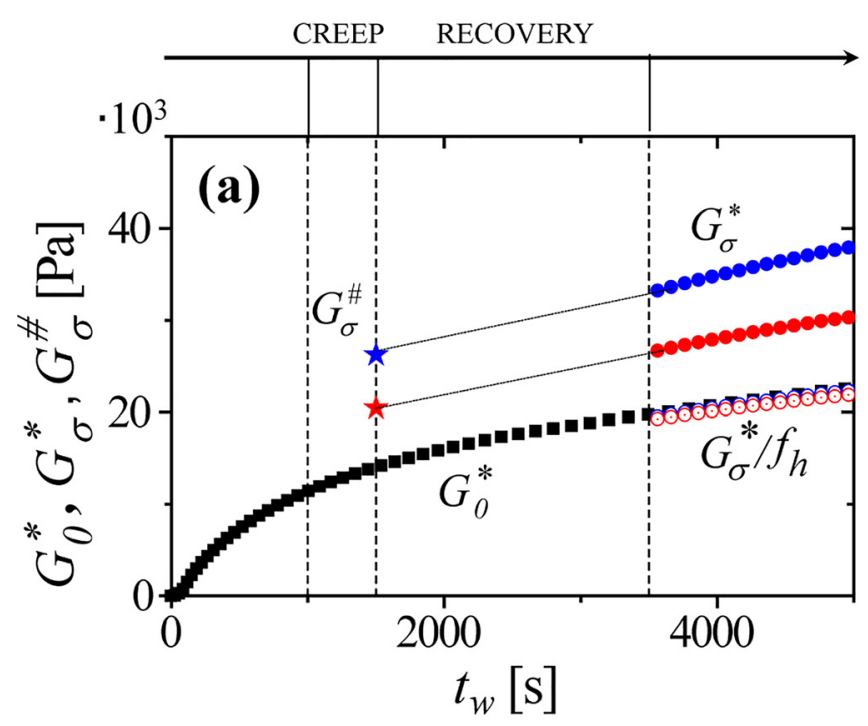

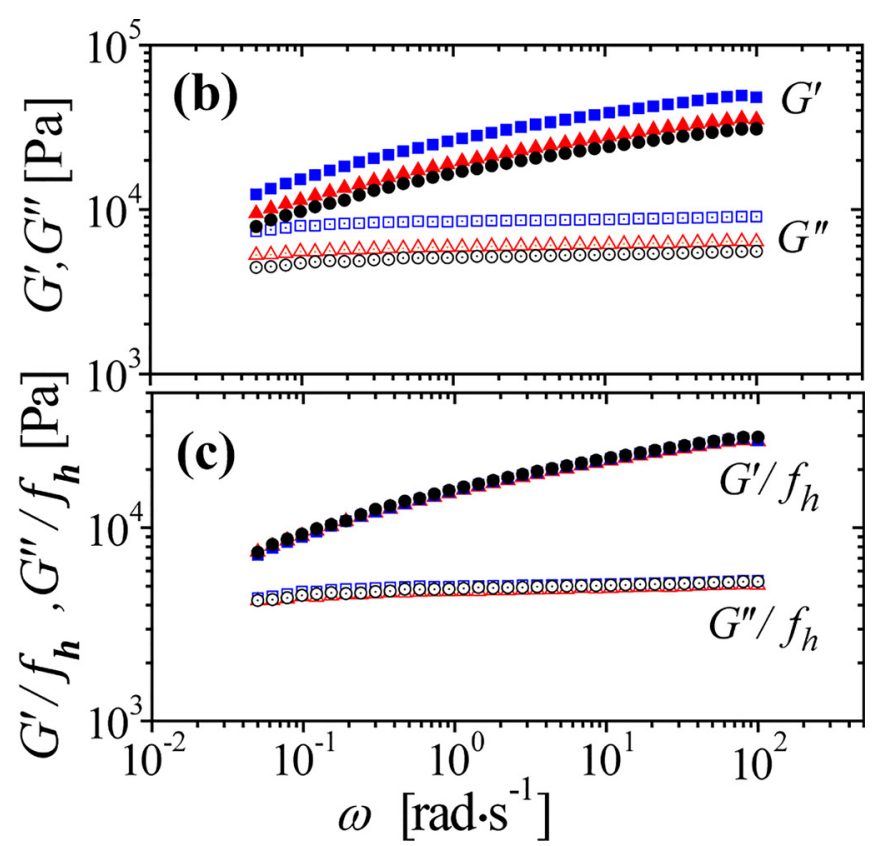

FIG. 5. Investigation of stress hardening. (a) Age dependence of complex modulus measured with $\omega=10 \mathrm{rad} / \mathrm{s}$ and $\gamma=10^{-3}$ for the quiescent gel $G_{0}^{*}$ and for the gels after creep and recovery $G_{\sigma}^{*}$. The stars denote $G_{\sigma}^{\#}=\sigma / \gamma_{I}$ determined from the instantaneous recovered strain after creep. Open symbols denote $G_{\sigma}^{*}$ normalized with a hardening factor $f_{h}=G_{\sigma}^{\#} / G_{0}^{*}$. (b) Frequency dependence of $G^{\prime}$ and $G^{\prime \prime}$ measured at $t_{w}=4000 \mathrm{~s}$ for the quiescent gel and for the gels after creep and recovery. (c) A simple normalization of the moduli obtained for the stress hardened gels with $f_{h}$ leads to a master curve. This shows that the application of stress leads to hardening, but does not alter the structural relaxation process.

structural reconfigurations on larger length scales. Despite such apparent differences in the processes governing the increase in elasticity and relaxation time, respectively, the rate of structural rearrangements nonetheless governs the rate at which the system hardens under stress. The data indicated as crosses in Fig. 6(b) are obtained for an age of $t_{w}=2000 \mathrm{~s}$ using a stress of $32 \mathrm{~Pa}$ for the creep experiment; $\left(f_{h}-1\right) / \sigma$ is here distinctly lower than for the experiment with $t_{w}=1000 \mathrm{~s}$. We can account for both, the age dependence of $f_{h}-1$ and the sublinear development of $f_{h}-1$ with $\tau$, by considering the decreasing rate of structural rearrangements due to aging, $1 / \tau_{R}\left(t_{w}\right)$. Defining the number of times the system has been restructured during the time interval of stress application as
$N_{R}=\int_{t_{w}}^{t_{w}+\tau}\left(1 / \tau_{R}\left(t_{w}\right)\right) d \tau$, we find that reporting $\left(f_{h}-1\right) / \sigma$ as a function of $N_{R}$ leads to a collapse of the age-dependent data and to a reasonable linearization of the lag-time dependence, as shown in Fig. 6(c). The description of our data by a unique linear dependence on $N_{R}$ suggests that stress hardening is not only controlled by stretching the network but also by the reconfiguration of the network triggered by thermal effects. This is remarkable considering (i) that stress hardening does not lead to a modification of the structural relaxation process itself and (ii) that the hardening effect is independent of whether we apply stresses that are below or above the threshold stress $\sigma_{c}$ defining the transition between pseudolinear and nonlinear creep denoted in Fig. 3(b).
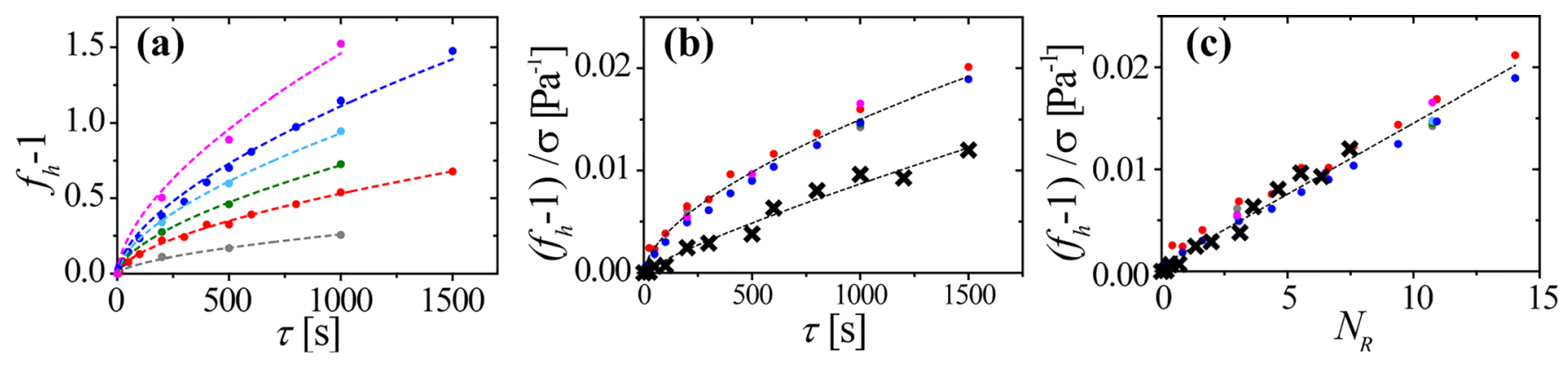

stress imposed during creep [Pa]

$$
\begin{aligned}
& \text { start of stress application at } t_{w}=1000 \mathrm{~s}: \quad 18 \bullet 32 \bullet 50 \bullet 64 \bullet 78 \bullet 92 \\
& \text { start of stress application at } t_{w}=2000 \mathrm{~s}: \quad \times 32
\end{aligned}
$$

FIG. 6. Investigation of the parameters governing stress hardening. (a) Reduced hardening factor $f_{h}-1$ as a function of the duration of stress applications starting at $t_{w}=1000 \mathrm{~s}$. Dashed lines are guides to the eye. (b) Normalization of $f_{h}-1$ with the stress imposed during creep leads to a collapse of the data sets reported in (a). Denoted as crosses are data obtained with $\sigma=32 \mathrm{~Pa}$ starting the stress application at $t_{w}=2000 \mathrm{~s}$. (c) Data shown in (b) as a function of the number of times $N_{R}$ the system has been restructured by thermal aging during stress application. All data can be described by a unique master curve displaying linear behavior. 


\section{Intermittent rearrangements leading to losses and delayed elastic contributions}

In contrast, the dependence of the lost strain $\gamma_{L}$ on $\sigma$ and $\tau$ exhibits distinct behavior below and above the threshold stress $\sigma_{c}$. As shown in Fig. 7, $\gamma_{L}$ depends on the duration of stress application, the magnitude of the stress applied during creep and the sample age. Unlike for the hardening factor, a normalization of $\gamma_{L}$ by $\sigma$ does not lead to a collapse of all the data obtained at a given sample age. As shown in Fig. 7(b), $\gamma_{L} / \sigma$ obtained for $t_{w}=1000 \mathrm{~s}$ displays a common development for $\sigma<\sigma_{c}$ but the data obtained at larger stresses exhibit a systematic stress dependence. This reflects the stress dependent response of the system beyond $\sigma_{c}$, which was depicted in the total compliance shown in Fig. 3(b). To assess the impact of the evolution of the viscosity on the lagtime dependence of $\gamma_{L}$ we consider the viscosity of the gel taking stress hardening into account. As discussed above, the relaxation time depends only on the age of the sample, while the elasticity depends on the sample age and, because of stress hardening, on the magnitude of the stress applied and the time interval over which the stress is applied. Thus, we redefine the viscosity as $\eta_{\sigma}=G_{\sigma}^{\#} \tau_{R}$ and calculate the expectation values of the loss contributions to the compliance as $J_{L}=\int_{t_{w}}^{t_{w}+\tau}\left(1 / \eta_{\sigma}\right) d \tau$. The parametric plots shown in Fig. 7(c) reveal that the evolution of $\gamma_{L}$ follows that of $J_{L}$ independent of whether the stress is below or above $\sigma_{c}$, as evidenced by the linear relation $\gamma_{L} / \sigma=a J_{L}$ obtained for all data sets. For $\sigma<\sigma_{c}$ the expectation values correspond approximately to those experimentally obtained $(a \approx 1)$; this includes the data obtained at $t_{w}=2000 \mathrm{~s}$ applying a stress of $32 \mathrm{~Pa}$ shown as crosses in Figs. 7(b) and 7(c). In contrast, for $\sigma>\sigma_{c}$, the experimentally obtained loss contributions to the compliance $\gamma_{L} / \sigma$ are significantly larger than expected $(a>1)$. Thus, imposing a stress larger than $\sigma_{c}$ leads to an increase of the loss contributions by a constant factor $a$, but not to a decoupling of the temporal evolution of $\gamma_{L} / \sigma$ from the intrinsic aging characteristics of the gel described by $J_{L}$.
This finding provides new insight regarding the temporal evolution of the parameters determining structural relaxations by intermittent rearrangements. As discussed previously, these parameters are (a) the rate of restructuring events occurring within the sample volume $\Gamma_{e}$ and (b) the volume of the rearranged zone per event $V_{e}$. The characteristic time reflecting the rearrangement of the entire sample volume $V_{s}$ is then $\tau_{R}=\left(\Gamma_{e} V_{e} / V_{s}\right)^{-1}$. That the temporal evolution of $\gamma_{L}$ remains correlated to the age dependence of $\tau_{R}$ for $\sigma>\sigma_{c}$, while the absolute magnitude of $\gamma_{L}$ is significantly larger than expected, suggests that only one of the two parameters, $\Gamma_{e}$ or $V_{e}$, is affected by increasing the stress beyond $\sigma_{c}$. This parameter should be age independent or almost age independent, as the correlation with the age dependence of $\tau_{R}$ would otherwise be lost due to a dominance of the stress-induced effects. If $\Gamma_{e}$ would remain constant during aging, $V_{e}$ would, just like $1 / \tau_{r}$, decrease with $t_{w}$. However, because of elastic coupling we would expect $V_{e}$ to rather increase than decrease with increasing $t_{w}$ due to the increase of the gel elasticity during aging. It is thus more likely that $\Gamma_{e}$ is the age-dependent and $V_{e}$ the ageindependent parameter. The age dependence of the relaxation time then solely reflects the age dependence of the restructuring rate $\tau_{R}\left(t_{w}\right) \propto 1 / \Gamma_{e}\left(t_{w}\right)$. That the absolute magnitude of $\gamma_{L}$ is larger than expected for $\sigma>\sigma_{c}$ is then consistent with the idea that imposing a stress larger than $\sigma_{c}$ leads to an increase of $V_{e}$, but does not affect $\Gamma_{e}\left(t_{w}\right)$, which remains that of the quiescent gel.

The remarkable linearization obtained for $\gamma_{L} / \sigma$ as a function $J_{L}$ shows that the deviations of the measured shear rates from the expectation values observed at shorter lag times in Fig. 3(d) must be due to delayed elasticity; the evolution of the loss contributions are described by the age-dependent viscosity over the entire lag-time window investigated, as shown in Fig. 7(b). Indeed, the delayed elastic contributions dominate the response function at short $\tau$ while the loss contributions become dominant at long times. This is shown in Fig. 8, where we display the lag-time dependence of $\gamma_{L}$ and
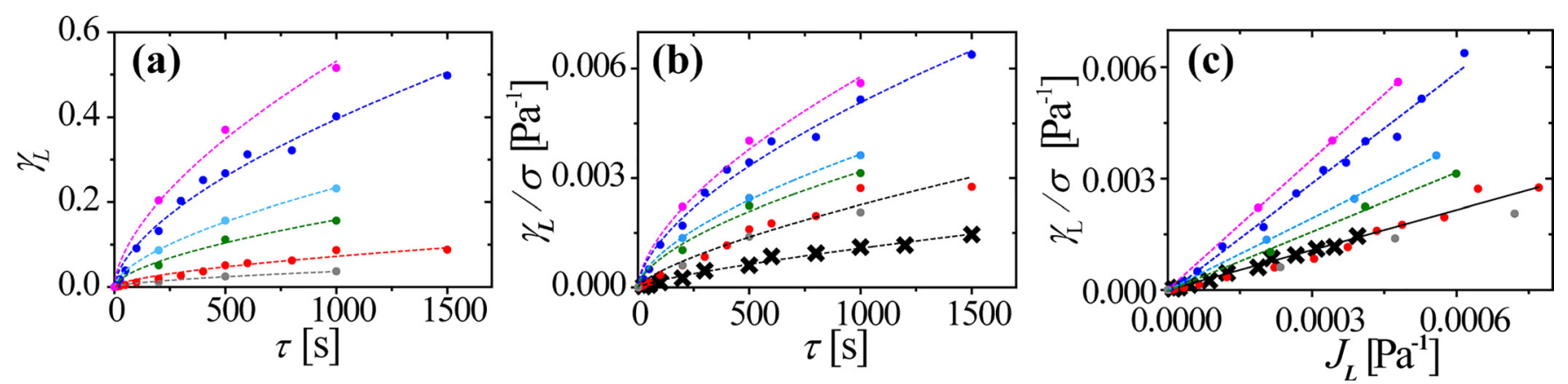

\author{
stress imposed during creep [Pa] \\ start of stress applicationat $t_{w}=1000 \mathrm{~s}: \quad 18 \bullet 32 \bullet 50 \bigcirc 64 \bullet 78 \bigcirc 92$ \\ start of stress application at $t_{w}=2000 \mathrm{~s}: \quad \times 32$
}

FIG. 7. Investigation of the parameters governing the loss contributions. (a) Irreversible, lost strain $\gamma_{L}$ as a function of the duration of stress applications starting at $t_{w}=1000 \mathrm{~s}$. Dashed lines are guides to the eye. (b) Normalization of $\gamma_{L}$ data shown in (a) with the stress imposed during creep. Only the data with $\sigma<\sigma_{c}$ display a common behavior. Denoted as crosses are data obtained with $\sigma=32 \mathrm{~Pa}$ starting the stress application at $t_{w}=2000 \mathrm{~s}$. (c) Graphing the data shown in (b) as a function of the expectation values for the compliance of the gel undergoing both thermal aging and stress hardening $J_{L}$ leads to the linearization of all data sets, $\gamma_{L} / \sigma=a \cdot J_{L}$. The data with $\sigma<\sigma_{c}$ display a common behavior with $a$ approximately unity. 

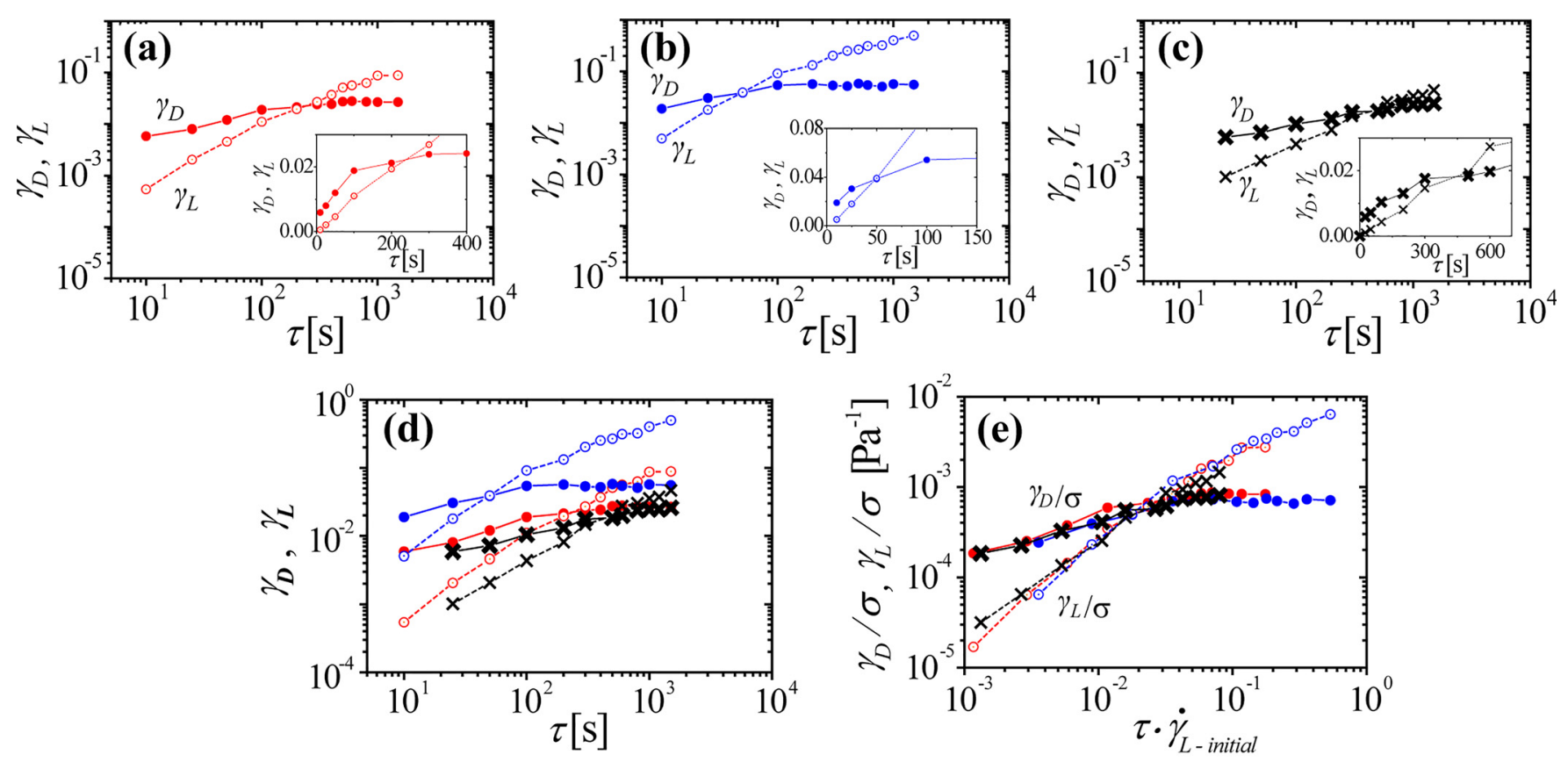

stress imposed during creep [Pa]

start of stress application at $t_{w}=1000 \mathrm{~s}$ : start of stress application at $\mathrm{t}_{\mathrm{w}}=2000 \mathrm{~s}$ :

- 32 - 78

$\times 32$

FIG. 8. Investigation of the parameters governing delayed elastic contributions $\gamma_{D}$ in comparison to the loss contributions $\gamma_{L} \cdot \gamma_{D}$ and $\gamma_{L}$ for: (a) A creep stress of $\sigma=32 \mathrm{~Pa}$ imposed at $t_{w}=1000 \mathrm{~s}$; (b) a creep stress of $\sigma=78 \mathrm{~Pa}$ imposed at $t_{w}=1000 \mathrm{~s}$; (c) a creep stress of $\sigma=32 \mathrm{~Pa}$ imposed at $t_{w}=2000 \mathrm{~s}$. Insets: Linear representation of $\gamma_{D}$ and $\gamma_{L}$ covering the short lag-time window. (d) and (e) The different data sets collapse on a common master curve when normalizing $\gamma_{D}$ and $\gamma_{L}$ with the stress imposed during creep and $\tau$ with the strain rate $\dot{\gamma}_{L-\text { initial }}$ defined as $d \gamma_{L} / d \tau$ in the limit of $\tau \rightarrow 0$.

$\gamma_{D}$ obtained for $t_{w}=1000 \mathrm{~s}$ and $\sigma=32$ and $78 \mathrm{~Pa}$, respectively, and for $t_{w}=2000 \mathrm{~s}$ and $\sigma=32 \mathrm{~Pa}$. The linear representation of the data shown in the insets reveals that the delayed elastic strain initially increases at approximately the same rate as the lost strain. Moreover, at the moment at which the lost strain starts to dominate the delayed elastic contribution starts to saturate. These characteristics indicate that delayed elastic storage relates to the process governing losses, namely, intermittent rearrangements.

To account for the evolution of the delayed elastic contributions, we use arguments similar to those developed for molecular glasses [70-72]. With no stress applied intermittent rearrangements occur in random directions. Upon imposing a small stress, however, the energy barriers are slightly biased, such that the relaxation events will favor the stress direction. The region rearranged will support less stress, such that the regions that are not yet rearranged will have to support more. Upon removal of the stress the matrix that has not undergone relaxation events will instantaneously recover, thereby imposing a local stress on the zones that have rearranged. To return to their initial configuration, these zones have to overcome again a local energy barrier, which leads to time delayed recovery. At early times, most of the relaxation events contribute to delayed elasticity; they turn into inelastic, permanently lost contributions only once the surrounding matrix has had enough time to be reconfigured by further rearrangement events. Because the gel integrity is maintained during restructuring the elastic loading process is constantly renewed, such that the delayed elastic contributions saturates when the lost contributions become dominant.
Remarkably, the magnitude of this saturation value is independent of whether the stress is below or above $\sigma_{\mathrm{c}}$. The rate at which $\gamma_{D}$ initially increases depends on the shear rate that becomes nonlinear for $\sigma>\sigma_{\mathrm{c}}$, but the $\gamma_{D}$ values obtained in the long-time limit of the experiment solely depend on stress. This is demonstrated in Fig. 8(e), where we normalize the time axis with the initial strain rate of the loss contributions, $\dot{\gamma}_{L-i n i t i a l}$, and the strain axis with the stress. This normalization leads to a collapse of the different data sets, consistent with the idea that the delayed elastic contributions are due to restructuration events prior to full structural relaxation. In our system, these restructuration events lead to a recoverable strain that is about a factor of ten larger than the instantaneously recovered strain; this suggests that a remarkably large fraction of the gel can be restructured before the integrity of the space spanning matrix is lost. Experiments exploring the recoverable strain of glassy systems denoted significantly smaller delayed elastic contributions as compared to the instantaneous one $[25,73,74]$, and it will be interesting to explore the parameters that set the magnitude of the delayed elastic contributions in future work.

\section{SUMMARY AND CONCLUSIONS}

We have explored the behavior of a colloidal gel that exhibits at least three evolving features: the rate of structural relaxation decreases in time, the elasticity increases in time, and the number of mobile particles decreases in time. Under stress the gel hardens at a faster rate than the quiescent gel, 
which is correlated to a faster decrease of the number of mobile particles. Such stress hardening does not impact the structural relaxation process, which indicates that the processes leading to an increase of, respectively, the elasticity and the structural relaxation time are not necessarily related. In support of this, we find that the structural relaxation process of our gel exhibits hyperaging similar to that reported in simulations of an attractive glass [58]. For this glass, the evolution of the structural relaxation and the bond-life time were found to be decoupled, consistent with our findings indicating that structural relaxations do not directly relate to particle mobility. We thus consider that we need to distinguish between two distinct processes, which we here tentatively term coarsening and aging. While coarsening involves the evolution of the structure due to the gradual incorporation of mobile particles into the network, aging is associated with intermittent events involving the rearrangement of ensembles of particles. Within the context of the features discussed above, we would thus presume that the gel elasticity increases mainly because of coarsening, while the structural relaxation relates mainly to aging.

The systematic investigation of the creep and recovery behavior of our gel allowed us to assess different contributions to creep, namely: an instantaneous elastic, a delayed elastic, and a loss contribution. The time dependence of all three contributions are determined by the evolving structural relaxation and elasticity denoted in the quiescent gel, where for the elasticity we have also to account for a stresshardening effect. Most remarkably, our data provide evidence that structural relaxations are due to the gradual restructuration of the gel by intermittent rearrangement events. In particular, the considerable delayed elastic contributions are consistent with the idea that the zones restructured during stress application experience a local stress once the macroscopic stress is removed, unless enough time has passed to restructure the entire gel by intermittent rearrangements. Upon approach of the yield stress, we find that the loss contributions increase by a constant factor, while their temporal evolution remains that expected from the aging characteristics of the quiescent gel including a stress-hardening effect. This suggests that upon approaching the yield conditions, the size of the zones rearranged per event increases, while the rate of events remains unchanged. Within the context of delayed yielding, we thus envision that there is a critical size of the intermittent rearrangements rather than a critical rate of restructuring events that defines the onset to yield. Further investigations are needed to fully explore this conjecture.

\section{ACKNOWLEDGMENTS}

The authors thank Emanuela Del Gado for fruitful discussions. Financial support from the Swiss National Science Foundation (Nos. 200021-157214 and 200020140908) is gratefully acknowledged.

\section{References}

[1] Liu, A. J., and S. R. Nagel, "The jamming transition and the marginally jammed solid," Annu. Rev. Condens. Matter Phys. 1, 347-369 (2010).
[2] Lu, P. J., and D. A. Weitz, "Colloidal particles: Crystals, glasses, and gels,” Annu. Rev. Condens. Matter Phys. 4, 217-233 (2013).

[3] Zaccarelli, E., "Colloidal gels: Equilibrium and non-equilibrium routes," J. Phys. Condens. Matter 19, 323101 (2007).

[4] Cates, M. E., M. Fuchs, K. Kroy, W. C. K. Poon, and A. M. Puertas, "Theory and simulation of gelation, arrest and yielding in attracting colloids,” J. Phys. Condens. Matter 16, S4861-S4875 (2004).

[5] Zia, R. N., B. J. Landrum, and W. B. Russel, "A micro-mechanical study of coarsening and rheology of colloidal gels: Cage building, cage hopping, and Smoluchowski's ratchet," J. Rheol. 58, 1121-1157 (2014).

[6] Conrad, J. C., H. M. Wyss, V. Trappe, S. Manley, K. Miyazaki, L. J. Kaufman, A. B. Schofield, D. R. Reichman, and D. A. Weitz, "Arrested fluid-fluid phase separation in depletion systems: Implications of the characteristic length on gel formation and rheology," J. Rheol. 54, 421-438 (2010).

[7] Lu, P. J., E. Zaccarelli, F. Ciulla, A. B. Schofield, F. Sciortino, and D. A. Weitz, "Gelation of particles with short-range attraction," Nature 453, 499-503 (2008).

[8] Manley, S., H. M. Wyss, K. Miyazaki, J. C. Conrad, V. Trappe, L. J. Kaufman, D. R. Reichman, and D. A. Weitz, "Glasslike arrest in spinodal decomposition as a route to colloidal gelation,” Phys. Rev. Lett. 95, 238302 (2005).

[9] Meakin, P., "Formation of fractal clusters and networks by irreversible diffusion-limited aggregation," Phys. Rev. Lett. 51, 1119-1122 (1983).

[10] Poon, W. C. K., A. D. Pirie, and P. N. Pusey, "Gelation in colloidpolymer mixtures," Faraday Discuss. 101, 65-76 (1995).

[11] Verhaegh, N. A. M., D. Asnaghi, H. N. W. Lekkerkerker, M. Giglio, and L. Cipelletti, "Transient gelation by spinodal decomposition in colloid-polymer mixtures," Physica A 242, 104-118 (1997).

[12] Weitz, D. A., and M. Oliveria, "Fractal structures formed by kinetic aggregation of aqueous gold colloids," Phys. Rev. Lett. 52, 1433-1436 (1984).

[13] Zaccarelli, E., P. J. Lu, F. Ciulla, D. A. Weitz, and F. Scortino, "Gelation as arrested phase separation in short-ranged attractive colloidpolymer mixtures," J. Phys. Condens. Matter 20, 494242 (2008).

[14] Puertas, A. M., M. Fuchs, and M. E. Cates, "Dynamical heterogeneities close to a colloidal gel," J. Chem. Phys. 121, 2813-2822 (2004).

[15] Puertas, A. M., M. Fuchs, and M. E. Cates, "Dynamical heterogeneities in an attraction driven colloidal glass," J. Non-Cryst. Solids 352, 4830-4834 (2006).

[16] Dibble, C. J., M. Kogan, and M. J. Solomon, "Structure and dynamics of colloidal depletion gels: Coincidence of transitions and heterogeneity," Phys. Rev. E 74, 041403 (2006).

[17] Dibble, C. J., M. Kogan, and M. J. Solomon, "Structural origins of dynamical heterogeneity in colloidal gels," Phys. Rev. E 77, 050401 (2008).

[18] Andrade, E. N. D., "On the viscous flow in metals, and allied phenomena," Proc. R. Soc. A 84, 1-12 (1910).

[19] Chen, M. W., "Mechanical behavior of metallic glasses: Microscopic understanding of strength and ductility," Annu. Rev. Mater. Res. 38, 445-469 (2008).

[20] Fielding, S. M., P. Sollich, and M. E. Cates, "Aging and rheology in soft materials," J. Rheol. 44, 323-369 (2000).

[21] Struik, L. C. E., Physical Aging in Amorphous Polymers and Other Materials (Elsevier Science Ltd, Amsterdam, 1978).

[22] El-Awady, J. A., "Unravelling the physics of size-dependent dislocation-mediated plasticity," Nat. Commun. 6, 5926 (2015).

[23] Miguel, M. C., A. Vespignani, M. Zaiser, and S. Zapperi, "Dislocation jamming and Andrade creep," Phys. Rev. Lett. 89, 165501 (2002). 
[24] Caton, F., and C. Baravian, "Plastic behavior of some yield stress fluids: From creep to long-time yield," Rheol. Acta 47, 601-607 (2008).

[25] Coussot, P., H. Tabuteau, X. Chateau, L. Tocquer, and G. Ovarlez, "Aging and solid or liquid behavior in pastes," J. Rheol. 50, 975-994 (2006).

[26] Grenard, V., T. Divoux, N. Taberlet, and S. Manneville, "Timescales in creep and yielding of attractive gels," Soft Matter 10, 1555-1571 (2014).

[27] Siebenburger, M., M. Ballauff, and T. Voigtmann, "Creep in colloidal glasses," Phys. Rev. Lett. 108, 255701 (2012).

[28] Lidon, P., L. Villa, and S. Manneville, "Power-law creep and residual stresses in a carbopol gel," Rheol. Acta 56, 307-323 (2016).

[29] Brenner, T., S. Matsukawa, K. Nishinari, and R. Johannsson, "Failure in a soft gel: Delayed failure and the dynamic yield stress," J NonNewtonian Fluid Mech. 196, 1-7 (2013).

[30] Gopalakrishnan, V., and C. F. Zukoski, "Delayed flow in thermoreversible colloidal gels," J. Rheol. 51, 623-644 (2007).

[31] Landrum, B. J., W. B. Russel, and R. N. Zia, "Delayed yield in colloidal gels: Creep, flow, and re-entrant solid regimes," J. Rheol. 60, $783-807$ (2016).

[32] Lindstrom, S. B., T. E. Kodger, J. Sprakel, and D. A. Weitz, "Structures, stresses, and fluctuations in the delayed failure of colloidal gels," Soft Matter 8, 3657-3664 (2012).

[33] Sprakel, J., S. B. Lindstrom, T. E. Kodger, and D. A. Weitz, "Stress enhancement in the delayed yielding of colloidal gels," Phys. Rev. Lett. 106, 248303 (2011).

[34] Dingenouts, N., C. Norhausen, and M. Ballauff, "Observation of the volume transition in thermosensitive core-shell latex particles by small-angle X-ray scattering," Macromolecules 31, 8912-8917 (1998).

[35] Fournet, G., and A. Guinier, Small Angle Scattering of X-Rays (John Wiley \& Sons, New York, 1955).

[36] Halperin, A., M. Kroger, and F. M. Winnik, "Poly(N-isopropylacrylamide) phase diagrams: Fifty years of research," Angew. Chem. Int. Ed. Engl. 54, 15342-15367 (2015).

[37] Guo, H. Y., S. Ramakrishnan, J. L. Harden, and R. L. Leheny, "Gel formation and aging in weakly attractive nanocolloid suspensions at intermediate concentrations," J. Chem. Phys. 135, 154903 (2011).

[38] Rueb, C. J., and C. F. Zukoski, "Viscoelastic properties of colloidal gels," J. Rheol. 41, 197-218 (1997).

[39] Zaccone, A., J. J. Crassous, and M. Ballauff, "Colloidal gelation with variable attraction energy," J. Chem. Phys. 138, 104908 (2013).

[40] Kantor, Y., and I. Webman, "Elastic properties of random percolating systems," Phys. Rev. Lett. 52, 1891-1894 (1984).

[41] DeRooij, R., D. Vandenende, M. H. G. Duits, and J. Mellema, "Elasticity of weakly aggregating polystyrene latex dispersions," Phys. Rev. E 49, 3038-3049 (1994).

[42] Romer, S., H. Bissig, P. Schurtenberger, and F. Scheffold, "Rheology and internal dynamics of colloidal gels from the dilute to the concentrated regime," Europhys. Lett. 108, 48006 (2014).

[43] Shih, W. H., W. Y. Shih, S. I. Kim, J. Liu, and I. A. Aksay, "Scaling behavior of the elastic properties of colloidal gels," Phys. Rev. A 42, 4772-4779 (1990).

[44] Weitz, D. A., and D. J. Pine, Dynamic Light Scattering (Oxford University, New York, 1993).

[45] Viasnoff, V., F. Lequeux, and D. J. Pine, "Multispeckle diffusingwave spectroscopy: A tool to study slow relaxation and timedependent dynamics," Rev. Sci. Instrum. 73, 2336-2344 (2002).

[46] Cipelletti, L., H. Bissig, V. Trappe, P. Ballesta, and S. Mazoyer, "Time-resolved correlation: A new tool for studying temporally heterogeneous dynamics," J. Phys. Condens. Matter 15, S257-S262 (2003).
[47] Duri, A., H. Bissig, V. Trappe, and L. Cipelletti, "Time-resolved-correlation measurements of temporally heterogeneous dynamics," Phys. Rev. E 72, 051401 (2005).

[48] Scheffold, F., S. E. Skipetrov, S. Romer, and P. Schurtenberger, "Diffusing-wave spectroscopy of nonergodic media," Phys. Rev. E 63, 061404 (2001).

[49] Krall, A. H., and D. A. Weitz, "Internal dynamics and elasticity of fractal colloidal gels," Phys. Rev. Lett. 80, 778-781 (1998).

[50] Cipelletti, L., L. Ramos, S. Manley, E. Pitard, D. A. Weitz, E. E. Pashkovski, and M. Johansson, "Universal non-diffusive slow dynamics in aging soft matter," Faraday Discuss. 123, 237-251 (2003).

[51] Crassous, J., and A. Saint-Jalmes, "Probing the dynamics of particles in an aging dispersion using diffusing wave spectroscopy," Soft Matter 8, 7683-7689 (2012).

[52] Snabre, P., and J. Crassous, "Multispeckle diffusing wave spectroscopy of colloidal particles suspended in a random packing of glass spheres," Eur. Phys. J. E 29, 149-155 (2009).

[53] Krall, A. H., Z. Huang, and D. A. Weitz, "Dynamics of density fluctuations in colloidal gels," Physica A 235, 19-33 (1997).

[54] Kamp, S. W., and M. L. Kilfoil, "Universal behaviour in the mechanical properties of weakly aggregated colloidal particles," Soft Matter $\mathbf{5}$, 2438-2447 (2009).

[55] Koumakis, N., and G. Petekidis, "Two step yielding in attractive colloids: Transition from gels to attractive glasses," Soft Matter 7, 2456-2470 (2011).

[56] Manley, S., B. Davidovitch, N. R. Davies, L. Cipelletti, A. E. Bailey, R. J. Christianson, U. Gasser, V. Prasad, P. N. Segre, M. P. Doherty, S. Sankaran, A. L. Jankovsky, B. Shiley, J. Bowen, J. Eggers, C. Kurta, T. Lorik, and D. A. Weitz, "Time-dependent strength of colloidal gels,” Phys. Rev. Lett. 95, 048302 (2005).

[57] Bissig, H., S. Romer, L. Cipelletti, V. Trappe, and P. Schurtenberger, "Intermittent dynamics and hyper-aging in dense colloidal gels," PhysChemComm 6, 21-23 (2003).

[58] Puertas, A. M., M. Fuchs, and M. E. Cates, "Aging in attraction-driven colloidal glasses,” Phys. Rev. E 75, 031401 (2007).

[59] Duri, A., and L. Cipelletti, "Length scale dependence of dynamical heterogeneity in a colloidal fractal gel," Europhys. Lett. 76, 972-978 (2006).

[60] Durian, D. J., D. A. Weitz, and D. J. Pine, "Multiple light-scattering probes of foam structure and dynamics," Science 252, 686-688 (1991).

[61] Chung, B., S. Ramakrishnan, R. Bandyopadhyay, D. Liang, C. F. Zukoski, J. L. Harden, and R. L. Leheny, "Microscopic dynamics of recovery in sheared depletion gels," Phys. Rev. Lett. 96, 228301 (2006).

[62] Cipelletti, L., S. Manley, R. C. Ball, and D. A. Weitz, "Universal aging features in the restructuring of fractal colloidal gels," Phys. Rev. Lett. 84, 2275-2278 (2000).

[63] Gao, Y., J. H. Kim, and M. E. Helgeson, "Microdynamics and arrest of coarsening during spinodal decomposition in thermoreversible colloidal gels," Soft Matter 11, 6360-6370 (2015).

[64] Teece, L. J., M. A. Faers, and P. Bartlett, "Ageing and collapse in gels with long-range attractions," Soft Matter 7, 1341-1351 (2011).

[65] Baravian, C., and D. Quemada, "Using instrumental inertia in controlled stress rheometry," Rheol. Acta 37, 223-233 (1998).

[66] Ewoldt, R. H., and G. H. McKinley, "Creep ringing in rheometry or how to deal with oft-discarded data in step stress tests!," Rheol. Bull. 76, 4-6 (2007).

[67] Divoux, T., C. Barentin, and S. Manneville, "From stress-induced fluidization processes to Herschel-Bulkley behaviour in simple yield stress fluids," Soft Matter 7, 8409-8418 (2011).

[68] Gibaud, T., D. Frelat, and S. Manneville, "Heterogeneous yielding dynamics in a colloidal gel," Soft Matter 6, 3482-3488 (2010). 
[69] Colombo, J., and E. Del Gado, "Stress localization, stiffening, and yielding in a model colloidal gel," J. Rheol. 58, 1089-1116 (2014).

[70] Argon, A. S., "Delayed elasticity in inorganic glasses," J. Appl. Phys. 39, 4080-4086 (1968).

[71] Gueguen, Y., V. Keryvin, T. Rouxel, M. Le Fur, H. Orain, B. Bureau, C. Boussard-Pledel, and J. C. Sangleboeuf, "A relationship between non-exponential stress relaxation and delayed elasticity in the viscoelastic process in amorphous solids: Illustration on a chalcogenide glass,” Mech. Mater. 85, 47-56 (2015).
[72] Trachenko, K., "Slow dynamics and stress relaxation in a liquid as an elastic medium," Phys. Rev. B 75, 212201 (2007).

[73] Petekidis, G., D. Vlassopoulos, and P. N. Pusey, "Yielding and flow of sheared colloidal glasses," J. Phys. Condens. Matter 16, S3955-S3963 (2004).

[74] Uhlherr, P. H. T., J. Guo, C. Tiu, X. M. Zhang, J. Z. Q. Zhou, and T. $\mathrm{N}$. Fang, "The shear-induced solid-liquid transition in yield stress materials with chemically different structures," J. Nonnewtonian Fluid. Mech. 125, 101-119 (2005). 